\title{
CARBON ISOTOPE MEASUREMENTS OF SURFACE SEAWATER FROM A TIME- SERIES SITE OFF SOUTHERN CALIFORNIA
}

\author{
Elise N Hinger ${ }^{1} \cdot$ Guaciara M Santos $•$ Ellen R M Druffel $\bullet$ Sheila Griffin \\ Earth System Science, Keck Carbon Cycle AMS Laboratory, University of California, Irvine, California 92697-3100, USA.
}

\begin{abstract}
We report carbon isotope abundances of dissolved inorganic carbon (DIC) in surface seawater collected from a time-series site off the Newport Beach Pier in Orange County, California. These data represent the first time series of $\Delta^{14} \mathrm{C}$ data for a coastal southern California site. From a suite of samples collected daily from 16 October to 11 November 2004, $\Delta^{14} \mathrm{C}$ values averaged $32.1 \pm 4.4 \%$. Freshwater input from the Santa Ana River to our site caused $\Delta^{14} \mathrm{C}$ and $\delta^{13} \mathrm{C}$ values to decrease. Since this initial set of measurements, a time-series site has been maintained from November 2004 to the present. Surface seawater has been collected bimonthly and analyzed for $\Delta^{14} \mathrm{C}, \delta^{13} \mathrm{C}$, salinity, and $\Sigma \mathrm{CO}_{2}$ concentrations. Water samples from the Santa Ana River were collected during the wet season. California sea mussels and barnacle shells, ranging from 4 to 6 months old, were also collected and analyzed. Results from May 2005 to January 2008 show no long-term changes in $\delta^{13} \mathrm{C}$ DIC values. $\Delta^{14} \mathrm{C}$ DIC values over the $2005-2006$ period averaged $33.7 \%$; high $\Delta^{14} \mathrm{C}$ values were observed sporadically (every 6-7 months), suggesting the presence of open water eddies at our site. Finally, in 2007, a stronger upwelling signal was apparent as indicated by correlations between $\Delta^{14} \mathrm{C}$, salinity, and the Bakun index, suggesting that the $\Delta^{14} \mathrm{C}$ record is an indicator of upwelling in the Southern California Bight.
\end{abstract}

\section{INTRODUCTION}

Dissolved inorganic carbon (DIC) is the largest pool of carbon in ocean water and is the precursor of organic matter produced by phytoplankton in the surface ocean. Interannual DIC $\Delta^{14} \mathrm{C}$ variability is primarily an indicator of ocean mixing, and is an indicator of upwelling in the open ocean (Ekman pumping; Guilderson et al. 2006) and coastal regions (Robinson 1981). Stable carbon isotope $\left(\delta^{13} \mathrm{C}\right)$ measurements of surface seawater and mollusks have also been used as a tracer of upwelling (Killingley and Berger 1979; Sheu et al. 1996).

The entire California coast is within one of the world's larger upwelling zones and its recurrent upwelling is responsible for stimulating marine productivity, fog formation, and also influencing the estuarine coastal habitats. We monitored DIC $\Delta^{14} \mathrm{C}$ at a coastal site off Southern California $\left(33^{\circ} 36^{\prime} 21^{\prime \prime} \mathrm{N}, 117^{\circ} 55^{\prime} 52^{\prime \prime} \mathrm{W}\right)$ where upwelling appears to vary seasonally (Masiello et al. 1998), to address the specific questions: 1) Is California coastal upwelling becoming weaker? 2) Is DIC $\Delta^{14} \mathrm{C}$ still a useful tracer for observing changes in upwelling? We analyzed seawater, shells, and local river water collected from our time-series site. We report a coupled isotope signature approach $\left(\Delta^{14} \mathrm{C}\right.$ and $\delta^{13} \mathrm{C}$ of DIC) combined with salinity and temperature measurements, total dissolved inorganic carbon $\left(\Sigma \mathrm{CO}_{2}\right)$ measurements, meteorological data, and ocean parameters to obtain a more accurate representation of the effects of ocean mixing on the carbon isotopic signatures of surface seawater from the coastal Orange County region (Figure 1).

\section{THE CALIFORNIA CURRENT FROM 1999 TO 2007}

Upwelling (the ascent of subsurface waters to the surface) can be observed in coastal and open ocean regions. Along the North American west coast, for example, upwelling occurs when winds combine with the Coriolis effect to produce an offshore Ekman transport of surface waters. The displaced coastal waters are replaced by cold, saline, nutrient-rich waters that upwell from depths of $50-100 \mathrm{~m}$ or more. Upwelled waters are generally characterized by lower temperature and higher salinity, chlorophyll $a$, and nutrient (nitrate, phosphate and silicate) concentrations (Hutchings et al. 1995).

${ }^{1}$ Corresponding author. Email: elisehinger@gmail.com. 


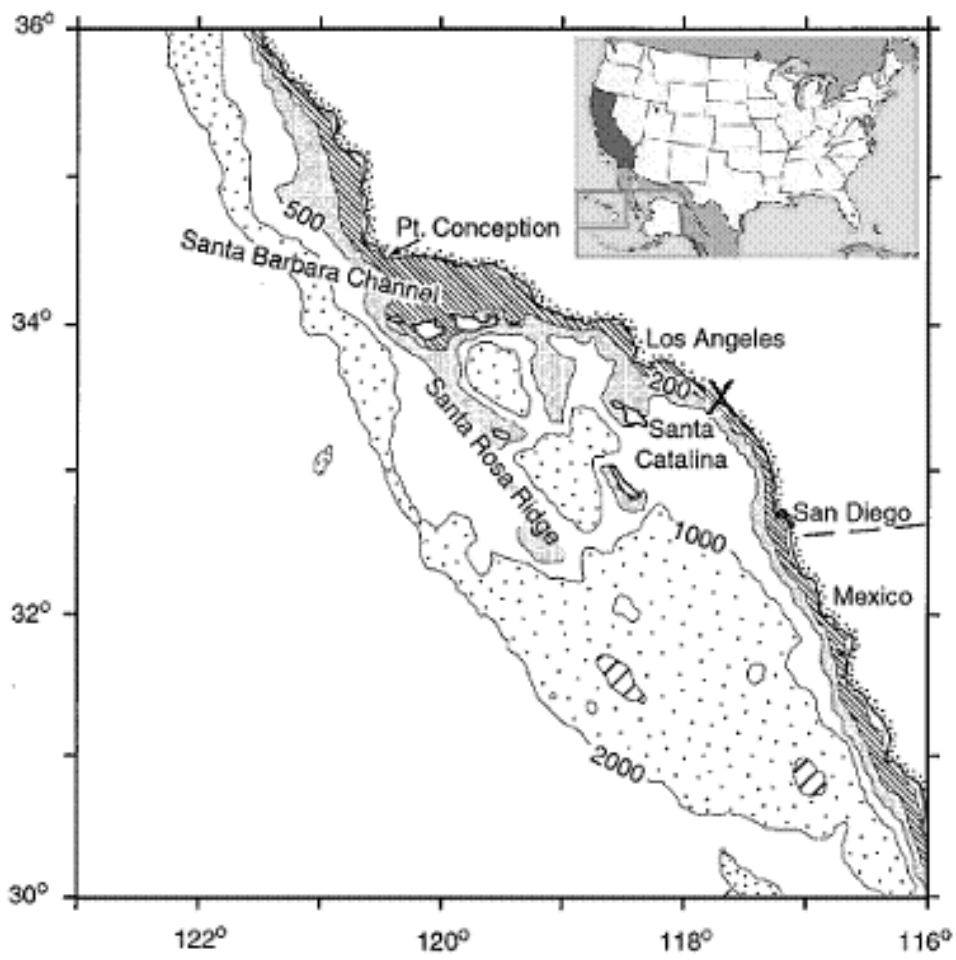

Figure 1 Water collection station off Orange County in the Southern California Bight, USA (indicated by X). Bathymetry is indicated by lines of constant depth in meters (after Bray et al. 1991).

Over the past $50 \mathrm{yr}$, several programs have monitored the meteorology, oceanography, chemistry, and biology of the California Current System (CCS) from Oregon to Baja California. In these programs, seasonal cruises reoccupy dozens of stations following grid lines from inshore to open waters. Overviews of the recent state of the CCS are presented in the data reports published by the California Cooperative Oceanic Fisheries Investigations (www.calcofi.org) (Peterson et al. 2006; Goericke et al. 2007), the Climate Prediction Center (http://www.cpc.ncep.noaa.gov), and the Northwest Fisheries Science Center (http://www.nwfsc.noaa.gov).

In general, the main observations provided by these programs along the North American west coast $\left(36^{\circ} \mathrm{N}\right.$ to $\left.57^{\circ} \mathrm{N}\right)$ show that from 1999 to 2002 , the CCS experienced strong summer upwelling and a shift to lower than normal sea surface temperatures (SSTs). During October 2002 to June 2005, weak El Niño conditions accompanied higher SSTs due to weak spring and summer upwelling south of Monterey Bay. In the Southern California Bight, SST anomalies were as high as $4{ }^{\circ} \mathrm{C}$ above normal (average seasonal temperature means from 1968-1996). In July to August 2005, upwelling of cold, saline, nutrient-rich water returned to the Southern California coast and continued through early 2006 (Peterson et al. 2006). Upwelling was weak through June 2006 and above average through fall (upwelling index anomalies were calculated relative to the 1948-1967 monthly means). In 2007, the CCS experienced near-to-above normal upwelling conditions from March to May and below average upwelling in June and July 2007 (Goericke et al. 2007). The cumulative upwelling for 2007 was above average with a transition to higher upwelling (La Niña phase) in late 2007 to early 2008 (http://www.cpc.ncep.noaa.gov). 


\section{THE SOUTHERN CALIFORNIA BIGHT}

The Southern California Bight (SCB) extends from Point Conception south to Cabo Colnett, Baja California (575 km), with the California Current System (CCS) to the west. The CCS flows southward, between California's shelf break to about $1000 \mathrm{~km}$ offshore. It is part of the clockwise geostrophic flow of water in the North Pacific gyre, carrying relatively cold, fresh subarctic water southward. The eastward indentation of the Southern California coast results in a counterclockwise gyre, known as the Southern California Eddy, in which water from the CCS is carried inshore and northward through the center of the bight to form the Southern California Countercurrent (Figure 2). Also within the SCB is the California Undercurrent, a narrow northward-flowing current of tropical origin; it flows between 100 and $400 \mathrm{~m}$ depth along the continental slope (Tsuchiya 1975). While a poleward flow of water predominates in the SCB due to the Southern California Countercurrent (surface flow) and California Undercurrent (subsurface flow), there are seasonal variations in surface water currents. The poleward surface flow of the Southern California Countercurrent is strongest during the summer months and continues through fall and winter. In spring, when equatorward winds along the southern California coast are strongest, the northward flow weakens or reverses, allowing an equatorward surface flow to prevail (Bray et al. 1991; PSCB 1990) (Figure 2).

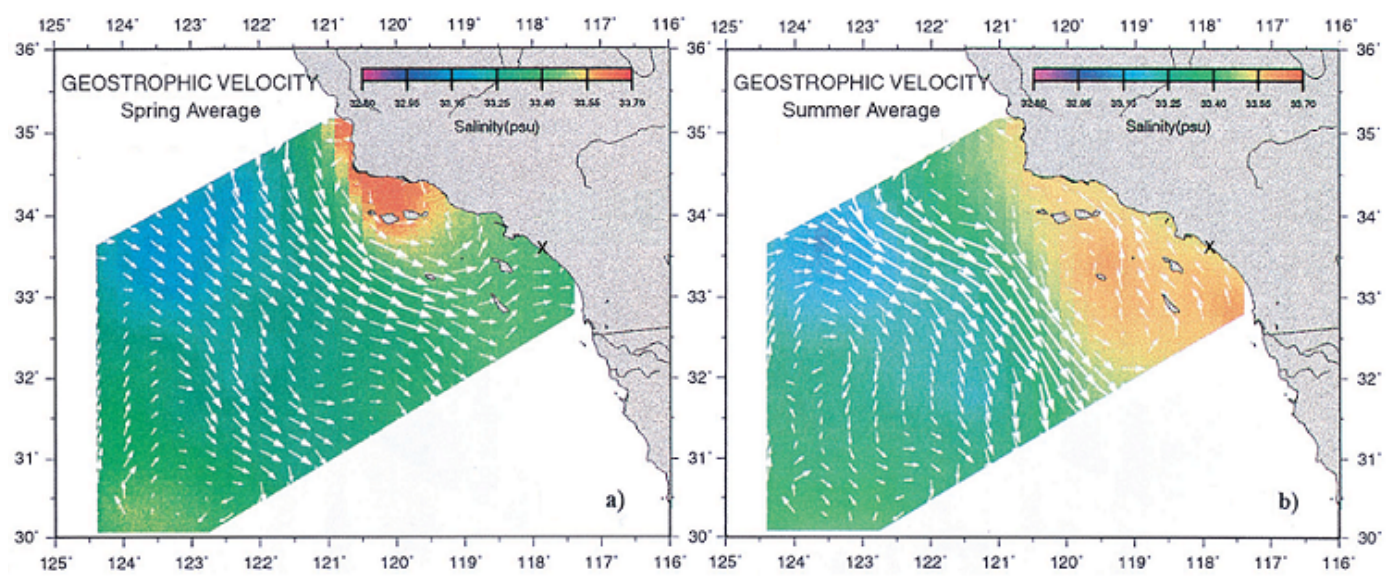

Figure 2 The average surface currents and salinity values in the Southern California Bight in (a) spring and (b) summer from 1984-1995. Northward flow along the coast is strongest in the summer months and weakens or reverses in the spring, allowing equatorward surface flow to prevail. Length of arrows represents relative geostrophic velocity. Color indicates salinity and "X" indicates our NBP sampling site. High salinities along the coast are associated with upwelling (Bray et al. 1991).

The sea floor of the bight is characterized by a series of deep basins (610-2500 $\mathrm{m}$ deep), islands, and submerged seamounts. The numerous basins, narrow continental shelf, and steep slopes throughout the SCB region allow deep water to exist near shore (Bray et al. 1991; PSCB 1990).

The SCB and its mainland drainage basin experience a semi-arid Mediterranean climate. Monthly precipitation near our sampling site peaks during the winter months, with a 5-yr range of $30-80 \mathrm{~mm}$, while summer months have near zero precipitation. Fall and spring months experience intermediate values of $0-40 \mathrm{~mm} / \mathrm{month}$ (http://waterdata.usgs.gov). This combination of semi-arid climate and low precipitation makes the rivers draining into the bight low flow.

Environmental changes in the SCB are linked to long-term, interannual patterns, such as El NiñoSouthern Oscillation (ENSO), rather than to seasonal cycles (Bray et al. 1991). The surface waters 
in the SCB originate primarily from the CCS and are therefore more nutrient-rich, less saline, and cooler, except when periodic ENSO events occur (PSCB 1990). ENSO events cause cooler surface waters to be replaced with nutrient-poor, warmer waters and a deeper surface mixed layer. Changes in weather and alterations in the marine community composition of the SCB can also be observed during ENSO events.

\section{SAMPLING SITES}

To determine a suitable sampling site for the coastal Orange County region in California, 4 seawater samples were collected, processed, and measured (for DIC $\Delta^{14} \mathrm{C}$, DIC $\delta^{13} \mathrm{C}$ ) from the shoreline, the Newport Beach Pier (NBP), and the Balboa Pier (BP) located 1.7 miles southeast of the NBP (Figure 3). The preliminary results indicated that these sites have similar isotopic compositions, and because of logistical considerations, the NBP site was chosen as our sampling site.

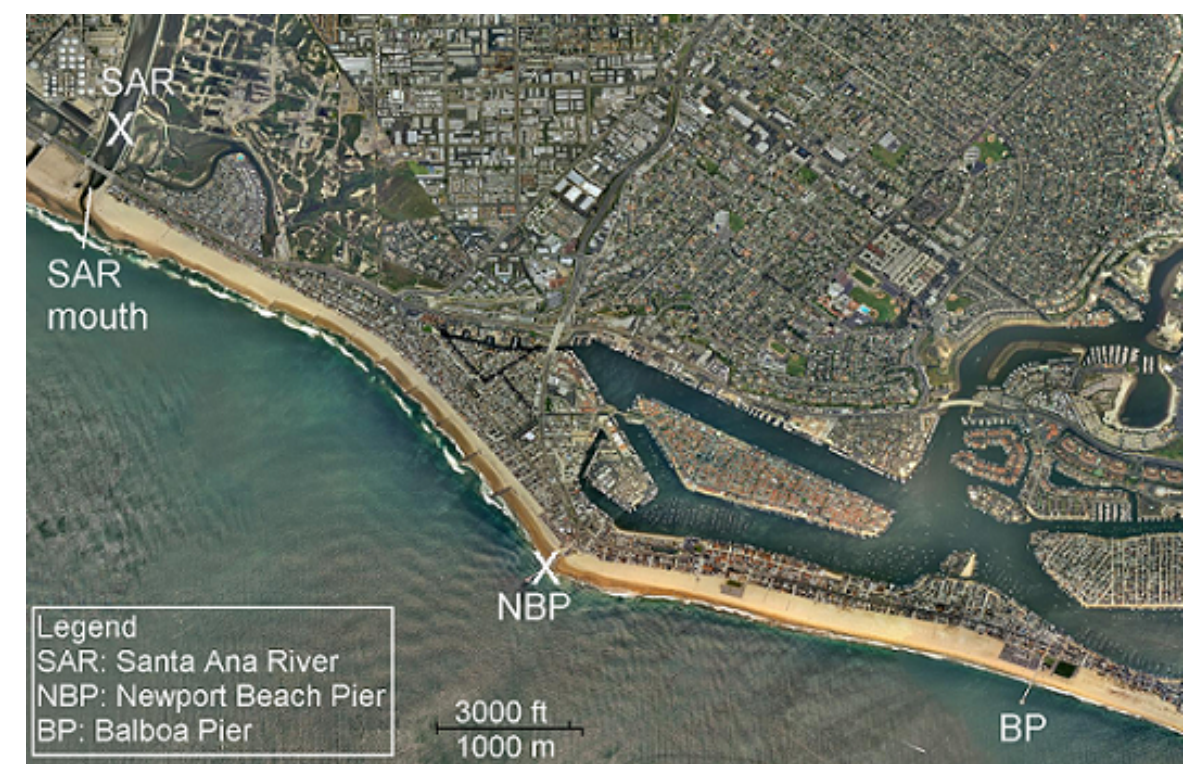

Figure 3 Satellite photo of the coastal region of Orange County. From left to right: the Santa Ana River mouth (SAR), the Newport Beach Pier (NBP) and the Balboa Pier (BP) are marked. These are the 3 sites from which samples were collected for this study (http://maps.google.com/).

The NBP site is located about 2 miles southeast of the mouth of the Santa Ana River. The Santa Ana River system is one of the largest rivers in Southern California with a watershed of $\sim 2800$ square miles (http://sawpa.org/about/watershed.htm). The river begins in the San Bernardino Mountains, travels through the Orange County Coastal Plain, and into the Pacific Ocean. Much of the Santa Ana River is a concrete-lined channel. It is characterized by near zero flow during the dry seasons and high surface flows through spring and early summer.

\section{METHODOLOGY}

\section{Water Sample Collection}

Surface seawater samples were collected daily from the NBP site from 16 October to 11 November 2004. Following this initial collection series, samples were collected biweekly between 10:00 AM 
and 1:00 PM (to assure the same tidal conditions). Surface waters were collected from a platform at the end of the pier that was lowered down to the water for sampling. Water samples were collected occasionally from the Santa Ana River at the Hamilton overpass, located $\sim 1.4$ miles upriver of the Santa Ana River mouth (Figure 3).

Two types of collection bottles were used. Seawater and river water samples for DIC $\Delta^{14} \mathrm{C}$ and $\delta^{13} \mathrm{C}$ analyses were collected in 500-mL glass bottles with ground glass stoppers. Samples analyzed for $\Sigma \mathrm{CO}_{2}$ and salinity were collected in $200-\mathrm{mL} \mathrm{Kimex}{ }^{\circledR}$ bottles with plastic caps. On 20 September 2007 , the sampling bottles used for $\Sigma \mathrm{CO}_{2}$ and salinity measurements were replaced with Pyrex ${ }^{\circledR} 250$ $\mathrm{mL}$ laboratory bottles with polypropylene caps. The previous collection bottles were found to be inferior because of evaporation of water from the samples.

Several precautions were taken to avoid contamination. Bottles and stoppers were washed using a dilute soap solution and water, followed by a $10 \% \mathrm{HCl}$ solution and distilled water. The glassware was baked at $550{ }^{\circ} \mathrm{C}$ for $2 \mathrm{hr}$ and stored in plastic bags.

Four samples were collected during each sampling: 2 for $\Delta^{14} \mathrm{C}$ analysis, 1 for $\Sigma \mathrm{CO}_{2}$, and 1 for salinity. At the sampling site, a 10-L bucket, equipped with a long piece of silicone tubing, was lowered into the water and rinsed several times prior to sampling. Each sample bottle was then filled with the tubing placed at the bottom of each bottle and allowed to overflow twice its volume. After all water samples were collected, the $\Delta^{14} \mathrm{C}$ and $\Sigma \mathrm{CO}_{2}$ samples were poisoned with $100 \mu \mathrm{L}$ and $50 \mu \mathrm{L}$ of saturated $\mathrm{HgCl}_{2}$ solution, respectively. The glass stoppers were coated with Apiezon $\mathrm{N}$ grease using a syringe to ensure a gas-tight seal. Each stopper was secured with 2 wide rubber bands placed around the cap and base of the bottle. After water collection, a thermometer (with an accuracy of $\pm 1{ }^{\circ} \mathrm{C}$ ) was placed in the remaining water and temperature was recorded along with other meteorological data, e.g. wind speed and direction, air temperature, recent precipitation events and possible river discharges, and water temperature measurements made by the lifeguards.

\section{Calcium Carbonate Collection}

Live California sea mussels (Mytilus californianus) and barnacle shells (spp. unknown) were collected from a support post of the pier, next to the water sampling site. These 2 species were selected due to their abundance in Southern California and availability at our sampling station. The first collection was performed on 4 May 2006 and the age of the mussel was estimated to be $\sim 6$ months old from its shell length of $\sim 35 \mathrm{~mm}$ (Coe and Fox 1942). A second mussel shell was collected on 18 June 2007 and its age was similarly $\sim 6$ months. On 31 January 2008, a third and fourth mussel were collected and estimated to be $\sim 5$ and $\sim 4$ months old, respectively.

\section{$\mathrm{CO}_{2}$ Extraction}

Water samples for $\Delta^{14} \mathrm{C}$ and $\delta^{13} \mathrm{C}$ analyses undergo $\mathrm{CO}_{2}$ extraction in the laboratory using a dedicated vacuum line. While 2 full bottles are collected for $\Delta^{14} \mathrm{C}$ analysis, only one-half bottle $(\sim 250 \mathrm{~mL})$ is needed per sample. This allows us to make up to 4 measurements of the same water to assess accuracy, if necessary.

The samples were stripped of $\mathrm{CO}_{2}$ using a modified extraction procedure of published protocols (McNichol and Jones 1991; McNichol et al. 1994). Half of the water in a DIC collection bottle was transferred into a custom reaction apparatus in a high-purity $\mathrm{N}_{2}$ glove bag. The original bottle that contained the rest of the water sample was resealed, reweighed, and stored for later use. The sample is connected to the vacuum line where it is acidified with $3 \mathrm{~mL}$ of $85 \%$ phosphoric acid and recirculated in the line with clean $\mathrm{N}_{2}$ for 10 min. Two dry ice-isopropanol slush traps collect water and a 
liquid nitrogen trap collects $\mathrm{CO}_{2}$. The $\mathrm{CO}_{2}$ is cryogenically purified and transferred to a calibrated volume for a pressure measurement, enabling us to determine the $\Sigma \mathrm{CO}_{2}$ of the sample.

The $\mathrm{CO}_{2}$ sample was split into 3 aliquots and flame-sealed into Pyrex tubes. Approximately 1.75 $\mathrm{mL}$ of $\mathrm{CO}_{2}$ gas was used to produce $\sim 1 \mathrm{mg} \mathrm{C}$ graphite for the $\Delta^{14} \mathrm{C}$ measurement, $0.54 \mathrm{~mL}$ for $\delta^{13} \mathrm{C}$ analysis, and the excess gas was archived.

Organic matter on the shell samples was separated from the calcite shell via microwaving and removal with a spatula. Dry, crushed calcium carbonate samples were placed in 3-mL Vacutainer ${ }^{\circledR}$ vials (disposable blood sample vials), leached using $2 \mathrm{~mL} 0.1 \mathrm{~N} \mathrm{HCl}$ to remove $50 \%$ of the sample's surface mass, and rinsed with deionized water. Vacutainers were then evacuated through the rubber stopper using a hypodermic needle connected to a vacuum line. Following evacuation, $0.8 \mathrm{~mL}$ of phosphoric acid was injected into each Vacutainer using a gas-tight syringe and hydrolyzed for 25 min at $70{ }^{\circ} \mathrm{C}$ to generate $\mathrm{CO}_{2}$ (Santos et al. 2004).

\section{Graphite Sample Production}

Carbon dioxide samples were converted to graphite by reduction with $\mathrm{H}_{2}$ in the presence of an iron powder catalyst at $550{ }^{\circ} \mathrm{C} . \mathrm{Mg}\left(\mathrm{ClO}_{4}\right)_{2}$ is present during the reaction for water removal (Santos et al. 2004, 2007). Following graphitization, samples are pressed into aluminum target holders that are mounted into the ion-source wheel and measured in the AMS system.

\section{$\Delta^{14} \mathrm{C}$ and $\delta^{13} \mathrm{C}$ Measurements}

All $\Delta^{14} \mathrm{C}$ and $\delta^{13} \mathrm{C}$ results (including freshwater and carbonate samples) obtained during this study are listed in Tables 1 and $2 . \Delta^{14} \mathrm{C}$ results were obtained at the KCCAMS/UCI facility, which is equipped with a compact AMS particle accelerator from National Electrostatics Corporation (NEC 0.5MV 1.5SDH-2 AMS system) dedicated to measuring ${ }^{14} \mathrm{C}$. This compact system measures all 3 carbon isotopes $\left({ }^{14} \mathrm{C},{ }^{13} \mathrm{C}\right.$, and ${ }^{12} \mathrm{C}$ ), allowing us to produce high-precision measurements (Santos et al. 2007). Each sample is corrected for fractionation using its own AMS $\delta^{13} \mathrm{C}$ value (which can differ by several per mil from the $\delta^{13} \mathrm{C}$ of the original material, if fractionation occurs during the AMS measurement). From general carbonaceous samples measured by this AMS system since 2002, the overall fractional scatter from the primary standard oxalic acid I (OX-I), as well as the accuracy based on the measurement of multiple secondary standards (OX-II, FIRI-C, FIRI-D, for example) has been $\sim 3 \%$ ( $\pm 1 \sigma)$ (Santos et al. 2007).

Table 1 Complete DIC $\Delta^{14} \mathrm{C}, \delta^{13} \mathrm{C}$, temperature, salinity, and $\Sigma \mathrm{CO}_{2}$ values from surface seawater samples collected from sites off the coast of Southern California. Calcium carbonate results are marked with asterisks. One result is from a freshwater sample from the Santa Ana River. The ${ }^{14} \mathrm{C}$ results are reported as age-corrected $\Delta^{14} \mathrm{C}(\%)$, as defined by Stuiver and Polach (1977). Total dissolved inorganic $\mathrm{CO}_{2}$ results were calculated from the manometric measurement of $\mathrm{CO}_{2}$ after acidification of seawater. Uncertainty for $\Sigma \mathrm{CO}_{2}$ values is $\pm 40 \mu \mathrm{mol} / \mathrm{kg}$.

\begin{tabular}{llllllll}
\hline $\begin{array}{l}\text { Sample ID } \\
\text { (dates in month/day/year) }\end{array}$ & $\begin{array}{l}\mathrm{Temp}^{\mathrm{a}} \\
\left({ }^{\circ} \mathrm{C}\right)\end{array}$ & $\begin{array}{l}\text { Salinity } \\
(\%)\end{array}$ & $\begin{array}{l}\text { Lab nr } \\
(\text { UCIAMS })\end{array}$ & $\begin{array}{l}\Delta^{14} \mathrm{C} \\
(\%)\end{array}$ & $\begin{array}{l}\delta^{13} \mathrm{C} \\
(\%)\end{array}$ & $\begin{array}{l}\Sigma \mathrm{CO}_{2} \\
(\mu \mathrm{mol} / \mathrm{kg})\end{array}$ \\
\hline 8/20/2004 Near NBP - shorec & & & 10407 & 37.8 & 2.9 & - & - \\
& & & 10408 & 32.8 & 2.4 & 1.6 & 1965 \\
& & & 10409 & 33.1 & 2.8 & - & 1959 \\
& & & 10410 & 35.7 & 2.9 & 1.6 & 1953 \\
8/20/2004 NBP Pierc & & & 10412 & 31.1 & 2.8 & 1.8 & 1948 \\
& & & 10413 & 35.2 & 2.9 & 1.7 & 1938 \\
& & & 10414 & 32.0 & 2.4 & 1.8 & 1937
\end{tabular}


Table 1 Complete DIC $\Delta^{14} \mathrm{C}, \delta^{13} \mathrm{C}$, temperature, salinity, and $\Sigma \mathrm{CO}_{2}$ values from surface seawater samples collected from sites off the coast of Southern California. Calcium carbonate results are marked with asterisks. One result is from a freshwater sample from the Santa Ana River. The ${ }^{14} \mathrm{C}$ results are reported as age-corrected $\Delta^{14} \mathrm{C}(\%)$, as defined by Stuiver and Polach (1977). Total dissolved inorganic $\mathrm{CO}_{2}$ results were calculated from the manometric measurement of $\mathrm{CO}_{2}$ after acidification of seawater. Uncertainty for $\Sigma \mathrm{CO}_{2}$ values is $\pm 40 \mu \mathrm{mol} / \mathrm{kg}$. (Continued)

\begin{tabular}{|c|c|c|c|c|c|c|c|}
\hline $\begin{array}{l}\text { Sample ID } \\
\text { (dates in month/day/year) }\end{array}$ & $\begin{array}{l}\text { Temp }{ }^{\mathrm{a}} \\
\left({ }^{\circ} \mathrm{C}\right)\end{array}$ & $\begin{array}{l}\text { Salinity } \\
(\%)\end{array}$ & $\begin{array}{l}\text { Lab nr } \\
\text { (UCIAMS) }\end{array}$ & $\begin{array}{l}\Delta^{14} \mathrm{C} \\
(\%)\end{array}$ & \pm & $\begin{array}{l}\delta^{13} \mathrm{C} \\
(\%)^{b}\end{array}$ & $\begin{array}{l}\Sigma \mathrm{CO}_{2} \\
(\mu \mathrm{mol} / \mathrm{kg})\end{array}$ \\
\hline \multirow[t]{3}{*}{ 9/22/2004 Balboa Pierc } & & & 13359 & 29.7 & 1.6 & 1.9 & 2101 \\
\hline & & & 13367 & 29.2 & 1.9 & 1.8 & 1951 \\
\hline & & & 13361 & 27.1 & 1.5 & 1.8 & 1946 \\
\hline \multirow{3}{*}{ 9/22/2004 NBP Pier } & & & 13358 & 32.7 & 1.9 & 1.7 & 1980 \\
\hline & & & 13360 & 31.9 & 1.7 & 1.7 & 1951 \\
\hline & & & 13362 & 32.2 & 1.7 & 1.8 & 1975 \\
\hline $10 / 16 / 2004$ & 19.8 & 33.34 & 13370 & 25.8 & 1.5 & 1.8 & 1995 \\
\hline $10 / 17 / 2004$ & 20.2 & 30.86 & 13371 & 37.8 & 1.5 & 1.3 & 1907 \\
\hline \multirow{3}{*}{$10 / 18 / 2004$} & 20.0 & 33.24 & 13356 & 35.2 & 1.7 & 1.6 & 1954 \\
\hline & & & 13357 & 32.9 & 1.6 & - & - \\
\hline & & & 13372 & 33.9 & 1.5 & 1.7 & 2012 \\
\hline $10 / 19 / 2004$ & 18.8 & 33.12 & 13373 & 35.5 & 1.5 & 1.7 & 2019 \\
\hline $10 / 20 / 2004$ & 19.6 & 33.17 & 13374 & 34.9 & 1.8 & 1.9 & 1974 \\
\hline \multirow[t]{2}{*}{$10 / 21 / 2004$} & 20.5 & 33.06 & 13375 & 37.7 & 1.5 & 1.5 & 1976 \\
\hline & & & 17053 & 31.6 & 1.7 & 1.5 & 2003 \\
\hline $10 / 22 / 2004$ & 19.4 & 32.87 & 13376 & 32.5 & 1.5 & 1.7 & 1607 \\
\hline $10 / 23 / 2004$ & 21.2 & 29.98 & 13377 & 32.3 & 1.6 & 0.1 & 1929 \\
\hline \multirow{2}{*}{$10 / 24 / 2004$} & 22.1 & 28.40 & 13378 & 20.9 & 1.7 & -0.5 & 2008 \\
\hline & & & 17054 & 23.6 & 1.5 & -1.1 & 2029 \\
\hline $10 / 25 / 2004$ & 24.0 & 28.89 & 13368 & 28.3 & 1.6 & 0.0 & 1959 \\
\hline $10 / 26 / 2004$ & 18.1 & 31.44 & 13380 & 32.4 & 1.6 & 1.6 & 1934 \\
\hline $10 / 27 / 2004$ & 19.5 & 32.87 & 13381 & 34.6 & 1.5 & 1.8 & 1969 \\
\hline $10 / 28 / 2004$ & 19.8 & 33.12 & 13382 & 31.0 & 2.0 & 1.7 & 1961 \\
\hline $10 / 29 / 2004$ & 20.0 & 33.05 & 13383 & 36.4 & 1.5 & 1.6 & 1986 \\
\hline \multirow[t]{2}{*}{$10 / 30 / 2004$} & 21.5 & 33.16 & 13384 & 26.8 & 1.6 & 1.5 & 1805 \\
\hline & & & 17055 & 29.9 & 1.6 & 1.5 & 1996 \\
\hline $10 / 31 / 2004$ & 20.5 & 33.06 & 13385 & 34.8 & 1.7 & 1.6 & 1988 \\
\hline $11 / 1 / 2004$ & 19.5 & 33.15 & 13386 & 33.3 & 1.6 & 1.4 & 2005 \\
\hline \multirow[t]{2}{*}{$11 / 2 / 2004$} & 19.5 & 33.17 & 13363 & 26.8 & 1.7 & 1.6 & 1987 \\
\hline & & & 17056 & 31.7 & 1.6 & 1.6 & 2002 \\
\hline $11 / 3 / 2004$ & 19.5 & 33.18 & 13364 & 33.0 & 1.5 & 1.8 & 1964 \\
\hline $11 / 4 / 2004$ & 19.4 & 33.08 & 13365 & 30.0 & 1.6 & 1.2 & 1946 \\
\hline \multirow[t]{2}{*}{$1 / 15 / 2005$} & 16.5 & 24.45 & 17057 & 21.0 & 1.5 & -2.1 & 1967 \\
\hline & & & 17058 & 22.7 & 2.5 & -1.9 & 1919 \\
\hline 2/9/2005 & 15.8 & 33.10 & 17059 & 32.0 & 1.5 & 1.3 & 1992 \\
\hline \multirow[t]{2}{*}{$2 / 12 / 2005$} & 16.2 & $\begin{array}{l}32.75 \\
32.85^{\mathrm{d}}\end{array}$ & 17060 & 34.4 & 1.9 & 1.5 & 1993 \\
\hline & & & 17061 & 31.6 & 1.5 & 1.5 & 1987 \\
\hline \multirow[t]{4}{*}{$2 / 23 / 2005$} & 16.8 & 31.86 & 17063 & 38.9 & 1.5 & 1.2 & 1976 \\
\hline & & & 17064 & 33.9 & 1.6 & 1.1 & 1976 \\
\hline & & & 22427 & 32.1 & 1.7 & - & - \\
\hline & & & 23542 & 38.7 & 1.5 & 1.1 & 1990 \\
\hline
\end{tabular}


Table 1 Complete DIC $\Delta^{14} \mathrm{C}, \delta^{13} \mathrm{C}$, temperature, salinity, and $\Sigma \mathrm{CO}_{2}$ values from surface seawater samples collected from sites off the coast of Southern California. Calcium carbonate results are marked with asterisks. One result is from a freshwater sample from the Santa Ana River. The ${ }^{14} \mathrm{C}$ results are reported as age-corrected $\Delta^{14} \mathrm{C}(\%)$, as defined by Stuiver and Polach (1977). Total dissolved inorganic $\mathrm{CO}_{2}$ results were calculated from the manometric measurement of $\mathrm{CO}_{2}$ after acidification of seawater. Uncertainty for $\Sigma \mathrm{CO}_{2}$ values is $\pm 40 \mu \mathrm{mol} / \mathrm{kg}$. (Continued)

\begin{tabular}{|c|c|c|c|c|c|c|c|}
\hline $\begin{array}{l}\text { Sample ID } \\
\text { (dates in month/day/year) }\end{array}$ & $\begin{array}{l}\text { Temp }{ }^{\mathrm{a}} \\
\left({ }^{\circ} \mathrm{C}\right)\end{array}$ & $\begin{array}{l}\text { Salinity } \\
(\%)\end{array}$ & $\begin{array}{l}\text { Lab nr } \\
\text { (UCIAMS) }\end{array}$ & $\begin{array}{l}\Delta^{14} \mathrm{C} \\
(\%)\end{array}$ & \pm & $\begin{array}{l}\delta^{13} \mathrm{C} \\
(\%)^{b}\end{array}$ & $\begin{array}{l}\Sigma \mathrm{CO}_{2} \\
(\mu \mathrm{mol} / \mathrm{kg})\end{array}$ \\
\hline \multirow{2}{*}{$3 / 11 / 2005$} & 17.5 & 32.54 & 17065 & 32.5 & 1.7 & 1.5 & 1985 \\
\hline & & & 17066 & 33.4 & 1.7 & 1.4 & 1980 \\
\hline \multirow{3}{*}{$3 / 25 / 2005$} & 17.0 & 32.59 & 17068 & 32.5 & 1.7 & 0.8 & 1977 \\
\hline & & & 22428 & 32.1 & 1.8 & - & - \\
\hline & & & 23543 & 34.8 & 1.6 & 0.6 & 2065 \\
\hline \multirow{2}{*}{$3 / 25 / 2005$ SAR } & - & 0.36 & 17070 & 1.5 & 1.5 & -11.3 & 2960 \\
\hline & & & 17071 & 0.3 & 1.7 & -11.3 & 2963 \\
\hline \multirow[t]{4}{*}{$4 / 15 / 2005$} & 17.0 & 32.44 & 17073 & 64.6 & 1.7 & 1.3 & 1960 \\
\hline & & & 17074 & 64.8 & 1.8 & - & - \\
\hline & & & 17075 & 65.9 & 1.8 & 1.3 & 1965 \\
\hline & & & 17076 & 66.3 & 2.4 & 1.4 & 1938 \\
\hline \multirow[t]{3}{*}{$5 / 9 / 2005$} & 19.8 & 33.01 & 17077 & 40.1 & 1.8 & 1.5 & 1988 \\
\hline & & & 17079 & 33.6 & 1.7 & 1.4 & 1969 \\
\hline & & & 22430 & 32.6 & 1.5 & - & - \\
\hline \multirow[t]{3}{*}{$5 / 27 / 2005$} & 19.5 & 33.44 & 17080 & 30.0 & 1.7 & 1.9 & 1959 \\
\hline & & & 22431 & 30.0 & 1.4 & - & - \\
\hline & & & 23544 & 33.3 & 1.7 & 2.0 & 1970 \\
\hline \multirow{3}{*}{$6 / 13 / 2005$} & 22.0 & 33.34 & 17082 & 34.8 & 1.6 & 2.6 & 1822 \\
\hline & & & 22433 & 31.8 & 1.5 & - & - \\
\hline & & & 23545 & 30.4 & 1.7 & 2.1 & - \\
\hline \multirow[t]{4}{*}{$6 / 27 / 2005$} & 19.5 & 33.41 & 22434 & 39.4 & 1.5 & 1.8 & 1962 \\
\hline & & & 22435 & 43.7 & 1.7 & 3.0 & 1963 \\
\hline & & & 23546 & 44.3 & 1.5 & 1.3 & 1878 \\
\hline & & & 23548 & 45.7 & 1.5 & - & 2003 \\
\hline \multirow[t]{2}{*}{$7 / 7 / 2005$} & 21.5 & $\begin{array}{l}33.49 \\
33.48^{\mathrm{d}}\end{array}$ & 22437 & 36.9 & 1.7 & 2.9 & 1803 \\
\hline & & & 22438 & 35.9 & 1.5 & 2.8 & 1812 \\
\hline \multirow{2}{*}{$7 / 21 / 2005$} & 24.5 & 33.45 & 22439 & 30.7 & 1.6 & - & 1828 \\
\hline & & & 22440 & 30.7 & 1.5 & 2.8 & 1839 \\
\hline 7/21/2005 SAR & - & 32.76 & 22441 & 42.2 & 1.6 & 1.8 & 2566 \\
\hline \multirow[t]{4}{*}{$8 / 4 / 2005$} & 21.5 & 33.32 & 22442 & 20.1 & 1.5 & 3.0 & 1789 \\
\hline & & & 22443 & 29.3 & 1.8 & 3.6 & 1743 \\
\hline & & & 23549 & 32.0 & 1.5 & 3.1 & - \\
\hline & & & 23550 & 32.6 & 1.6 & - & 1772 \\
\hline \multirow[t]{2}{*}{$8 / 18 / 2005$} & 21.0 & 33.32 & 22444 & 29.6 & 2.5 & 2.7 & 1847 \\
\hline & & & 23551 & 31.0 & 1.6 & & - \\
\hline \multirow[t]{2}{*}{$9 / 1 / 2005$} & 23.0 & 33.27 & 22446 & 29.9 & 1.9 & 2.4 & 1911 \\
\hline & & & 22447 & 31.5 & 1.5 & 2.6 & 1897 \\
\hline \multirow[t]{2}{*}{ 9/19/2005 } & 20.5 & 33.31 & 22448 & 39.2 & 1.5 & 2.6 & 1860 \\
\hline & & & 22449 & 38.8 & 1.5 & 2.4 & 1892 \\
\hline \multirow{2}{*}{$10 / 7 / 2005$} & 19.2 & 33.33 & 22450 & 35.0 & 1.7 & 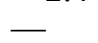 & 1937 \\
\hline & & & 22451 & 32.0 & 1.6 & 2.0 & 1804 \\
\hline
\end{tabular}


Table 1 Complete DIC $\Delta^{14} \mathrm{C}, \delta^{13} \mathrm{C}$, temperature, salinity, and $\Sigma \mathrm{CO}_{2}$ values from surface seawater samples collected from sites off the coast of Southern California. Calcium carbonate results are marked with asterisks. One result is from a freshwater sample from the Santa Ana River. The ${ }^{14} \mathrm{C}$ results are reported as age-corrected $\Delta^{14} \mathrm{C}(\%)$, as defined by Stuiver and Polach (1977). Total dissolved inorganic $\mathrm{CO}_{2}$ results were calculated from the manometric measurement of $\mathrm{CO}_{2}$ after acidification of seawater. Uncertainty for $\Sigma \mathrm{CO}_{2}$ values is $\pm 40 \mu \mathrm{mol} / \mathrm{kg}$. (Continued)

\begin{tabular}{|c|c|c|c|c|c|c|c|}
\hline $\begin{array}{l}\text { Sample ID } \\
\text { (dates in month/day/year) }\end{array}$ & $\begin{array}{l}\text { Temp }{ }^{\mathrm{a}} \\
\left({ }^{\circ} \mathrm{C}\right)\end{array}$ & $\begin{array}{l}\text { Salinity } \\
(\%)\end{array}$ & $\begin{array}{l}\text { Lab nr } \\
\text { (UCIAMS) }\end{array}$ & $\begin{array}{l}\Delta^{14} \mathrm{C} \\
(\%)\end{array}$ & \pm & $\begin{array}{l}\delta^{13} \mathrm{C} \\
(\%)^{\mathrm{b}}\end{array}$ & $\begin{array}{l}\Sigma \mathrm{CO}_{2} \\
(\mu \mathrm{mol} / \mathrm{kg})\end{array}$ \\
\hline & & & 22452 & 31.6 & 1.6 & - & - \\
\hline & & & 22453 & 36.3 & 1.8 & 2.0 & 1950 \\
\hline \multirow{2}{*}{$10 / 21 / 2005$} & 19.0 & 33.25 & 23552 & 31.7 & 1.5 & - & 1916 \\
\hline & & & 23553 & 35.8 & 1.4 & 2.0 & 1936 \\
\hline \multirow{2}{*}{$11 / 4 / 2005$} & 19.0 & 33.30 & 22454 & 33.7 & 1.7 & 2.1 & 1905 \\
\hline & & & 23554 & 26.9 & 1.8 & 1.4 & 2167 \\
\hline $11 / 18 / 2005$ & 18.5 & 33.29 & 23555 & 31.1 & 1.5 & 1.7 & 1942 \\
\hline \multirow{2}{*}{$12 / 2 / 2005$} & 16.5 & 33.29 & 23556 & 36.1 & 1.6 & 1.6 & 1962 \\
\hline & & & 31143 & 37.3 & 1.4 & 1.6 & 1974 \\
\hline $12 / 16 / 2005$ & 15.5 & 33.39 & 23557 & 33.5 & 1.4 & 1.2 & 2023 \\
\hline \multirow{2}{*}{$12 / 29 / 2005$} & 17.5 & 33.27 & 23558 & 36.6 & 1.4 & 1.4 & 1997 \\
\hline & & & 31144 & 33.5 & 1.4 & 1.3 & 1968 \\
\hline \multirow{2}{*}{$1 / 13 / 2006$} & 16.3 & 33.24 & 23559 & 49.9 & 2.0 & 1.5 & 1951 \\
\hline & & & 31145 & 52.2 & 1.4 & 1.6 & 1947 \\
\hline $2 / 2 / 2006$ & 15.5 & 33.40 & 23561 & 41.7 & 1.7 & 1.7 & 1961 \\
\hline $2 / 16 / 2006$ & 14.0 & 33.42 & 23560 & 41.1 & 1.5 & 3.3 & 1966 \\
\hline $3 / 2 / 2006$ & 17.0 & 33.22 & 23562 & 34.3 & 1.5 & 1.7 & 1968 \\
\hline $3 / 20 / 2006$ & 13.0 & 33.40 & 43656 & 25.8 & 1.3 & 0.8 & 2039 \\
\hline \multirow[t]{3}{*}{$4 / 6 / 2006$} & 15.0 & 30.34 & 31146 & 28.1 & 1.3 & -0.8 & 2064 \\
\hline & & & 31147 & 29.7 & 1.3 & -0.7 & 2087 \\
\hline & & & 31148 & 27.5 & 1.3 & - & 2075 \\
\hline $4 / 20 / 2006$ & 17.0 & 33.43 & 31149 & 27.8 & 1.6 & 1.3 & 1942 \\
\hline \multirow[t]{2}{*}{$5 / 4 / 2006$} & 18.5 & 32.82 & 31150 & 27.3 & 1.4 & 1.3 & 1948 \\
\hline & & & 43657 & 26.2 & 1.3 & 1.2 & 1907 \\
\hline 5/4/2006 Mussel Shell* & & & 31151 & 34.8 & 1.3 & - & - \\
\hline 5/4/2006 Barnacle Shell* & & & 31152 & 36.3 & 1.3 & - & - \\
\hline $5 / 18 / 2006$ & 18.0 & 33.50 & 31153 & 24.9 & 1.3 & 1.6 & 1954 \\
\hline $6 / 1 / 2006$ & 18.5 & 33.45 & 31154 & 27.8 & 1.3 & 2.2 & 1900 \\
\hline \multirow[t]{2}{*}{$6 / 15 / 2006$} & 18.0 & 33.42 & 31155 & 34.3 & 1.9 & 1.5 & 1986 \\
\hline & & & 31156 & 31.1 & 1.3 & 1.3 & 2009 \\
\hline $7 / 1 / 2006$ & 17.0 & 33.46 & 31157 & 37.7 & 1.4 & 1.2 & 2014 \\
\hline $7 / 15 / 2006$ & 19.5 & 33.43 & 31158 & 31.3 & 1.3 & 1.6 & 1995 \\
\hline \multirow[t]{2}{*}{$7 / 29 / 2006$} & 25.0 & 33.52 & 31159 & 28.9 & 1.3 & 1.9 & 1963 \\
\hline & & & 43658 & 31.3 & 1.8 & 2.0 & 1894 \\
\hline \multirow[t]{3}{*}{$8 / 15 / 2006$} & 20.0 & 33.40 & 31160 & 45.0 & 1.6 & 1.9 & 1969 \\
\hline & & & 43659 & 47.1 & 1.5 & 1.8 & 1975 \\
\hline & & & - & - & - & 1.9 & - \\
\hline $8 / 29 / 2006$ & 19.0 & 33.44 & 31161 & 33.0 & 1.4 & 1.6 & 1983 \\
\hline $9 / 14 / 2006$ & 19.0 & 33.41 & 31162 & 32.2 & 1.4 & 1.6 & 2022 \\
\hline $9 / 28 / 2006$ & 18.5 & 33.40 & 31163 & 30.9 & 1.4 & 1.5 & 1990 \\
\hline $10 / 17 / 2006$ & 19.0 & 33.39 & 31164 & 33.7 & 1.4 & 1.6 & 1976 \\
\hline $11 / 8 / 2006$ & 19.0 & 33.34 & 31165 & 32.9 & 1.4 & 1.7 & 1959 \\
\hline
\end{tabular}


Table 1 Complete DIC $\Delta{ }^{14} \mathrm{C}, \delta^{13} \mathrm{C}$, temperature, salinity, and $\Sigma \mathrm{CO}_{2}$ values from surface seawater samples collected from sites off the coast of Southern California. Calcium carbonate results are marked with asterisks. One result is from a freshwater sample from the Santa Ana River. The ${ }^{14} \mathrm{C}$ results are reported as age-corrected $\Delta^{14} \mathrm{C}(\%)$, as defined by Stuiver and Polach (1977). Total dissolved inorganic $\mathrm{CO}_{2}$ results were calculated from the manometric measurement of $\mathrm{CO}_{2}$ after acidification of seawater. Uncertainty for $\Sigma \mathrm{CO}_{2}$ values is $\pm 40 \mu \mathrm{mol} / \mathrm{kg}$. (Continued)

\begin{tabular}{|c|c|c|c|c|c|c|c|}
\hline $\begin{array}{l}\text { Sample ID } \\
\text { (dates in month/day/year) }\end{array}$ & $\begin{array}{l}\text { Temp }{ }^{\mathrm{a}} \\
\left({ }^{\circ} \mathrm{C}\right)\end{array}$ & $\begin{array}{l}\text { Salinity } \\
(\%)\end{array}$ & $\begin{array}{l}\text { Lab nr } \\
\text { (UCIAMS) }\end{array}$ & $\begin{array}{l}\Delta^{14} \mathrm{C} \\
(\%)\end{array}$ & \pm & $\begin{array}{l}\delta^{13} \mathrm{C} \\
(\%)^{b}\end{array}$ & $\begin{array}{l}\Sigma \mathrm{CO}_{2} \\
(\mu \mathrm{mol} / \mathrm{kg})\end{array}$ \\
\hline $11 / 22 / 2006$ & 19.0 & 33.35 & - & - & - & - & - \\
\hline $12 / 28 / 2006$ & 14.5 & 33.03 & 43660 & 32.1 & 1.4 & 1.5 & 2036 \\
\hline $1 / 11 / 2007$ & 14.0 & 33.54 & 43661 & 33.7 & 1.3 & 1.2 & 1971 \\
\hline 1/11/2007 SAR/ocean mixture & - & 24.81 & 43662 & 12.2 & 1.3 & -4.8 & 2734 \\
\hline $1 / 26 / 2007$ & 15.0 & 33.58 & 43663 & 36.7 & 1.4 & 1.3 & 1973 \\
\hline $2 / 14 / 2007$ & 15.0 & 33.40 & 43664 & 48.4 & 1.3 & 0.7 & 2010 \\
\hline $3 / 17 / 2007$ & 14.5 & 33.60 & 43665 & 29.9 & 1.7 & 1.4 & 1921 \\
\hline \multirow[t]{2}{*}{$4 / 2 / 2007$} & 15.0 & & 43666 & 27.6 & 1.3 & 1.0 & 1969 \\
\hline & & & 45399 & 27.7 & 2.1 & - & - \\
\hline \multirow[t]{2}{*}{$4 / 18 / 2007$} & 15.0 & 33.67 & 43667 & 26.4 & 1.3 & 1.2 & 1952 \\
\hline & & & 45400 & 27.8 & 2.5 & - & - \\
\hline $5 / 3 / 2007$ & 17.0 & - & 43668 & 22.1 & 1.3 & 1.1 & 2020 \\
\hline $5 / 18 / 2007$ & 15.5 & 33.76 & 43669 & 20.9 & 1.3 & 2.1 & 1884 \\
\hline $5 / 31 / 2007$ & 17.0 & 33.79 & 43670 & 22.7 & 1.3 & 2.1 & 1824 \\
\hline $6 / 18 / 2007$ & 18.0 & 33.75 & 43671 & 25.3 & 1.6 & 1.3 & 1995 \\
\hline \multirow[t]{2}{*}{ 6/18/2007 Mussel Shell* } & & & 43681 & 36.4 & 1.5 & - & - \\
\hline & & & 45410 & 32.3 & 2.3 & - & - \\
\hline $7 / 5 / 2007$ & 22.0 & 33.74 & 43672 & 25.7 & 1.3 & 1.8 & 1926 \\
\hline $7 / 20 / 2007$ & 20.0 & - & 43673 & 26.2 & 1.3 & 1.6 & 1926 \\
\hline $8 / 6 / 2007$ & 23.0 & - & 43674 & 23.1 & 1.3 & 2.1 & 1900 \\
\hline $8 / 21 / 2007$ & 22.0 & $\begin{array}{l}33.69 \\
33.69^{d}\end{array}$ & 43675 & 27.1 & 1.3 & 2.0 & 1918 \\
\hline $9 / 6 / 2007$ & 20.5 & 33.64 & 43676 & 28.9 & 1.6 & 1.7 & 1946 \\
\hline $9 / 20 / 2007$ & 19.0 & 33.63 & 43677 & 27.6 & 1.3 & 1.6 & 1979 \\
\hline $10 / 5 / 2007$ & 18.0 & 33.53 & 43678 & 29.6 & 1.3 & 1.5 & 1921 \\
\hline $10 / 19 / 2007$ & 17.0 & 33.45 & 43679 & 30.4 & 1.3 & 1.5 & 1943 \\
\hline $11 / 6 / 2007$ & 17.0 & $\begin{array}{l}33.52 \\
33.52^{\mathrm{d}}\end{array}$ & 43680 & 27.0 & 1.3 & 1.8 & 1913 \\
\hline $11 / 20 / 2007$ & 15.5 & 33.55 & 45401 & 29.6 & 2.1 & 1.6 & 1952 \\
\hline $12 / 6 / 2007$ & 16.0 & 33.44 & 45402 & 25.0 & 2.2 & 1.3 & 1951 \\
\hline $12 / 28 / 2007$ & 13.0 & 33.39 & 45403 & 29.5 & 2.2 & 1.4 & 1974 \\
\hline $1 / 4 / 2008$ & 14.0 & 33.43 & 45404 & 29.1 & 2.1 & 1.4 & 1989 \\
\hline $1 / 31 / 2008$ & 14.0 & $\begin{array}{l}33.42 \\
33.42^{\mathrm{d}}\end{array}$ & 45405 & 31.2 & 2.3 & 1.5 & 1974 \\
\hline 1/31/2008 Mussel Shell A* & & & 45411 & 29.1 & 2.2 & - & - \\
\hline 1/31/2008 Mussel Shell B* & & & 45412 & 27.4 & 2.2 & - & - \\
\hline
\end{tabular}

${ }^{a}$ Temperature measured from the remaining water mass after sampling.

${ }^{\mathrm{b}} \delta^{13} \mathrm{C}$ values measured using continuous-flow stable isotope ratio mass spectrometer (Finnigan Delta-Plus CFIRMS).

${ }^{\text {cPreliminary }}{ }^{14} \mathrm{C}$ measurements to help on the selection of the sampling station.

${ }^{\mathrm{d}}$ Duplicate measurements performed on the same water mass to evaluate procedural error. 
To obtain high-precision measurements on wheels composed of DIC samples, we added to the usual sets of 6-8 OX-I primary standards: OX-II samples, 2 to 3 DIC procedural blanks, and secondary coral standards produced in the same fashion as unknown samples (Table 2). Individual targets were cycled at least 12 times, and analyzed for $50,000{ }^{14} \mathrm{C}$ events. The individual statistical error bars from these samples were typically $\pm 2 \%$ o $( \pm 1 \sigma)$ (Tables 1 and 2 ). These errors were calculated based on counting statistics and scatter in multiple measurements of each sample, along with propagated uncertainties from normalization to standards, background subtraction, and isotopic fractionation corrections, as mentioned above.

Table 2 Complete DIC $\Delta{ }^{14} \mathrm{C}$ and $\delta^{13} \mathrm{C}$ values from blanks and secondary standards used to background-correct and evaluate precision and accuracy of the results produced. Calcium carbonate blank and secondary standard are marked with asterisks. In addition, batches of $\mathrm{CO}_{2} \mathrm{DIC}$ samples to be measured by the stable isotope ratio mass spectrometer were also followed by $\mathrm{CO}_{2}$ aliquots of OX-I (oxalic acid I) produced from a large amount of gas to verify the procedural error of this instrument (results not shown). The standard deviation of these measurements was $0.09 \%$ and standard error was $0.02 \%$ o $(n=32)$.

\begin{tabular}{llllllll}
\hline & $n$ & $\begin{array}{l}\mathrm{Fm}^{\mathrm{a}} \\
\text { average }\end{array}$ & $\begin{array}{l}\mathrm{Fm} \\
\text { st. error }\end{array}$ & $\begin{array}{l}\Delta^{14} \mathrm{C}(\%)^{\mathrm{b}} \\
\text { average }\end{array}$ & $\begin{array}{l}\Delta^{14} \mathrm{C}(\%) \\
\text { st. error }\end{array}$ & $\begin{array}{l}\delta^{13} \mathrm{C} \\
\text { average }^{\mathrm{c}}\end{array}$ & $\begin{array}{l}\delta^{13} \mathrm{C} \\
\text { st. error }^{\mathrm{d}}\end{array}$ \\
\hline DIC CSTD $^{\mathrm{e}}$ & 21 & 0.9440 & 0.0004 & -62.0 & 0.35 & 0.57 & 0.013 \\
DIC Calcite $^{\mathrm{f}}$ & 12 & 0.0065 & 0.0008 & -993.6 & 0.75 & 1.60 & 0.025 \\
Calcite $^{*}$ & 4 & 0.0017 & 0.0003 & -998.3 & 0.27 & - & - \\
FIRI-C $^{\mathrm{h}^{*}}$ & 4 & 0.1036 & 0.0002 & -897.3 & 0.18 & - & - \\
\hline
\end{tabular}

${ }^{\mathrm{a} F r a c t i o n}$ modern of carbon (Stuiver and Polach 1977).

${ }^{\mathrm{b} 14} \mathrm{C}$ values expressed as ${ }^{14} \mathrm{C}$, (Stuiver and Polach 1977)

${ }^{\mathrm{c}} \delta^{13} \mathrm{C}$ values measured using continuous-flow stable isotope ratio mass spectrometer (Finnigan Delta-Plus CFIRMS).

${ }^{\mathrm{d}}$ Procedural error based on number of replicates.

${ }^{\text {e }}$ Dissolved coral standard produced in previously stripped seawater samples.

${ }^{\mathrm{f}}$ Dissolved mineral calcite produced in previously stripped seawater samples to determinate procedural background.

gSolid mineral calcite.

hFIRI-C marine turbidite, consensus value of 18,176 yr BP (Scott et al. 2004).

For proper DIC $\Delta^{14} \mathrm{C}$ background corrections, we measured the mass and the $\Delta^{14} \mathrm{C}$ AMS signature of several procedural blank samples (Table 2). To produce these samples, $\sim 60 \mathrm{mg}$ of spar calcite crystals $\left({ }^{14} \mathrm{C}\right.$-free material with initial $\Delta{ }^{14} \mathrm{C}$ value of $-1000 \%$ ) were dissolved into a previously stripped seawater sample (and therefore free of DIC) in the presence of a high-purity $\mathrm{N}_{2}$ environment. In this case, the $\Delta^{14} \mathrm{C}$ result obtained from the procedural blank represents the amount of modern carbon added to the sample during $\mathrm{CO}_{2}$ stripping, graphitization, and pressing of graphite samples. The $\Delta^{14} \mathrm{C}$ AMS results from the DIC procedural blank averaged $-993.6 \%$ (equivalent to $\mathrm{Fm}=$ 0.0065 ; Table 2). This result constitutes a modern carbon contamination of $<0.8 \%$ of the sample, and was subtracted from the final DIC $\Delta{ }^{14} \mathrm{C}$ results listed in Table 1.

Stable isotope DIC $\delta^{13} \mathrm{C}$ measurements were made using a continuous-flow isotope ratio mass spectrometer (Delta-Plus CFIRMS), interfaced with a Gasbench II and dual inlet (for $\mathrm{CO}_{2}$ analysis). For standard control, the precision of the equipment was also evaluated by sets of $\mathrm{CO}_{2}$ gas from OX-I combustion produced and measured at the same time as unknown samples. The standard deviation of these measurements was $0.09 \%$ o $(n=32$, not shown in Table 2$)$.

To verify the experimental error associated with the DIC sample procedure, we analyzed multiple samples from the same sampling date (Table 1), in addition to the DIC blanks and coral standard 
samples (Table 2). In terms of reproducibility, the DIC $\Delta^{14} \mathrm{C}$ measurements are within the stated precision of $\pm 2.0 \% \mathrm{o}( \pm 1 \sigma)$, based on a pooled standard deviation calculation (McNaught and Wilkinson 1997) of the 39 sets of replicated measurements (Tables 1 and 2). The results obtained for DIC $\delta^{13} \mathrm{C}$ through CFIRMS measurements yielded a pooled standard deviation of $\pm 0.2 \%$ o $( \pm 1 \sigma ; 38$ data points). Since the nominal or statistical propagated errors from individual measurements (as shown on Tables 1 and 2) are mostly smaller than these calculated experimental uncertainties, we have chosen to use the larger errors during our discussion.

Calcium carbonate samples measured with the AMS system were background-corrected with the proper blank and checked by secondary standard samples (calcite crystals and FIRI-C marine turbidite, respectively) and their results are shown in Tables 1 and 2 (marked with asterisks).

\section{Temperature, Salinity, and $\Sigma \mathrm{CO}_{2}$}

Temperature, salinity, and $\Sigma \mathrm{CO}_{2}$ values from surface seawater are shown on Table 1. Temperature measurements were taken in situ. The temperature values obtained were later compared with the values obtained by the Newport Beach lifeguards from the same station as well as a Southern California Coastal Ocean Observing System (http://www.sccoos.org) automated station off Balboa Pier (Figure 4). The measurements made by the Newport Beach lifeguards are performed daily using a thermometer (with accuracy of $\pm 0.1^{\circ} \mathrm{F}$ ) placed at $\sim 3 \mathrm{~m}$ depth (from mean tide level) below the surface water. Temperature measurements obtained using these 3 methods show slight differences, but show the same general trend. Our temperature measurements are made in the upper $1 \mathrm{~m}$, while the other 2 measurements are made at $3 \mathrm{~m}$ depth. All temperature values used in the Discussion and Results section are our measurements.

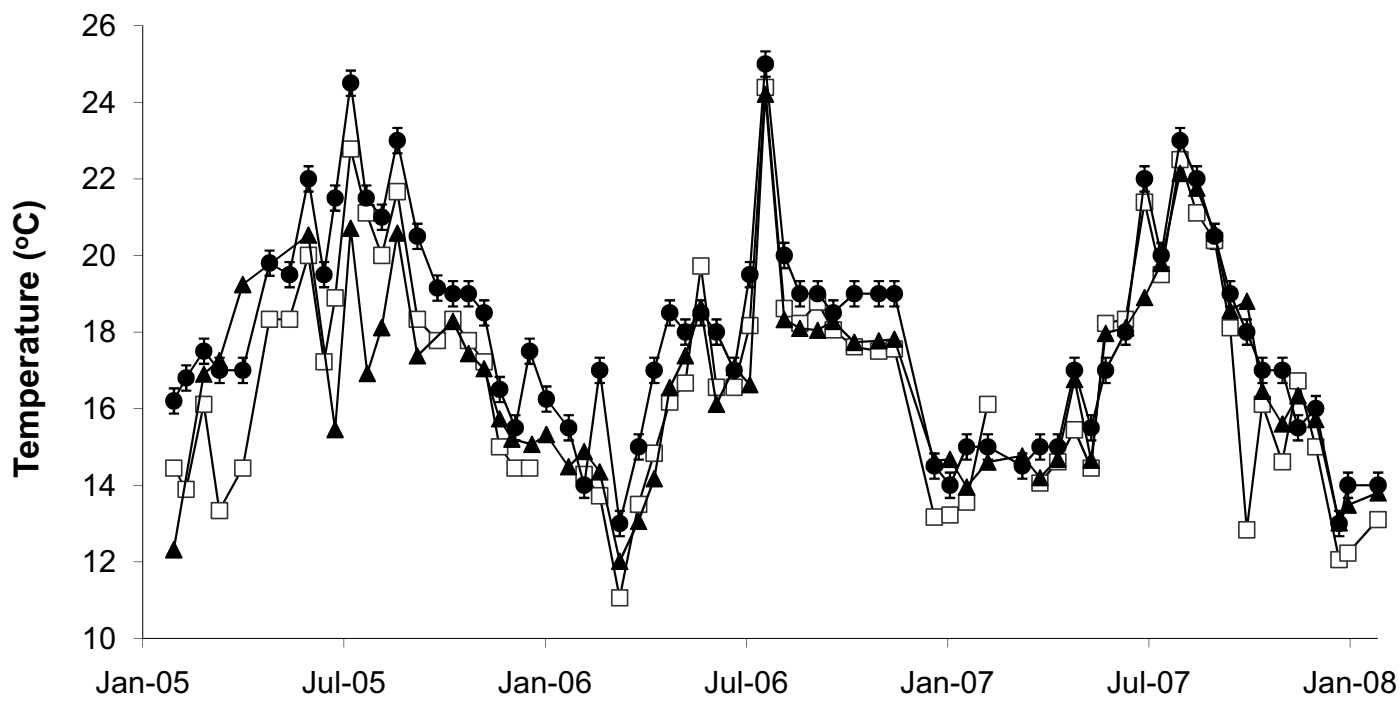

Figure 4 Temperature results obtained by 3 distinct proxies: ( $\square$ ) from NBP at $\sim 3 \mathrm{~m}$ depth, depending on the tidal conditions, recorded by the lifeguards; $(\bullet)$ from the NBP in surface seawater samples collected in this work; and ( $\mathbf{\Lambda})$ from Balboa Pier at $\sim 3 \mathrm{~m}$ depth obtained by the Southern California Coastal Observation System (SCCOOS) database (http://www.sccoos.org/).

Salinity measurements of seawater were made by the Scripps Oceanographic Data Facility (http:// sts.ucsd.edu/sts/chem) using a Guildline Autosal 8400A salinometer (nominal accuracy of $0.002 \%$ ). To determine measurement precision, 1 unknown sample per group submitted was 
selected to be remeasured, yielding a standard error calculation of $0.04 \%$ o $( \pm 1 \sigma)$, based on 5 pairs (Table 1).

$\Sigma \mathrm{CO}_{2}$ values were calculated from the manometric measurement of $\mathrm{CO}_{2}$ after the acidification of seawater. Initially, the $\Sigma \mathrm{CO}_{2}$ manometric calculation assumed a room temperature of $20{ }^{\circ} \mathrm{C}$ (italicized values in Table 1). Beginning with samples from December 2006, actual room temperature was recorded during the $\mathrm{CO}_{2}$ extraction of each water sample. The recorded temperature was taken into account for the $\Sigma \mathrm{CO}_{2}$ manometric calculation resulting in a more accurate value. The maximum error for these measurements is approximately $\pm 40 \mu \mathrm{mol} / \mathrm{kg}$.

\section{RESULTS AND DISCUSSION}

\section{NBP Daily Collection (October-November 2004)}

Figures $5 \mathrm{a}-\mathrm{f}$ show data obtained from temperature, salinity, $\Sigma \mathrm{CO}_{2}$, DIC $\Delta{ }^{14} \mathrm{C}$, and $\delta^{13} \mathrm{C}$ measurements from the NBP site, as well as Santa Ana River discharge data (obtained from the US Geological Survey database) from 10/15/04 to 11/11/04. The lines in Figures 5c-e represent the 3-point moving average. This experiment was designed to determine the daily variability of the carbon isotopic ratios and other chemical and meteorological parameters at this coastal site.

The SST values averaged $20.0^{\circ} \mathrm{C}$ with higher values (up to $24.0{ }^{\circ} \mathrm{C}$ ) from $10 / 23 / 04-10 / 25 / 04$ (Figure $5 \mathrm{a})$. Salinity values averaged $33.16 \%$, with lower values $(28.40-32.87 \%$ o) in samples collected on 10/17/04 and 10/23/04-10/26/04 due to admixture with Santa Ana River water (Figure 5b,f). $\Sigma \mathrm{CO}_{2}$ values averaged $1975 \mu \mathrm{mol} / \mathrm{kg}$ and showed no significant trend (Figure $5 \mathrm{c}$ ). The $\Delta^{14} \mathrm{C}$ values ranged from $20.9 \%$ to $37.8 \%$, with an average value of $32.1 \%$ (Figure $5 \mathrm{~d}$ ). The $\delta^{13} \mathrm{C}$ values ranged from $-1.1 \%$ to $+1.9 \%$ with an average value of $1.6 \%$ and a distinct low from $10 / 23 / 04-10 / 25 / 04$ (Figure 5e).

Three precipitation events occurred during the NBP Daily Collection series (Figure 5f). On 10/17/ 04 , a rain event $(12 \mathrm{~mm})$ resulted in a decreased salinity of 30.86\% at our site. On 10/20/04, a second rain event $(50 \mathrm{~mm})$ resulted in high Santa Ana River discharge from 10/20/04-10/25/04 and salinity values as low as $28.40 \%$ at our site. A third rain event occurred on 10/26/04-10/27/04 (31 $\mathrm{mm}$ ), resulting in high Santa Ana River runoff from 10/27/04-10/30/04. However, the salinity at our site remained constant at $33.10 \%$ during this period, indicating that the runoff from the third event did not reach our sampling site.

Analysis of wind patterns (not shown in Figure 5) during this period explains the lack of a third lower salinity signal. Typical winds at our site are of southwest origin (perpendicular to our NW-SE running coast) that strengthen in the afternoon and weaken in the evening. However, there were strong southerly winds (10-20 mph) on 10/26/04 and then easterly winds (10-15 mph) on 10/27/04. These atypical winds coincided with the third rain event and most likely pushed Santa Ana River water towards the northwest, away from our collection site. During typical conditions (that occurred throughout the rest of October 2004), winds from the southwest would decrease the off-coast movement of Santa Ana River runoff, causing the freshwater to spread along the coastline.

Freshwater input from the Santa Ana River also appears to have caused lower than average DIC $\Delta^{14} \mathrm{C}$ and $\delta^{13} \mathrm{C}$ values along with increased SST. When local river input is not present, salinity averaged $33.14 \%$. We found that salinity measurements were essential to detect the presence of freshwater at our site. 


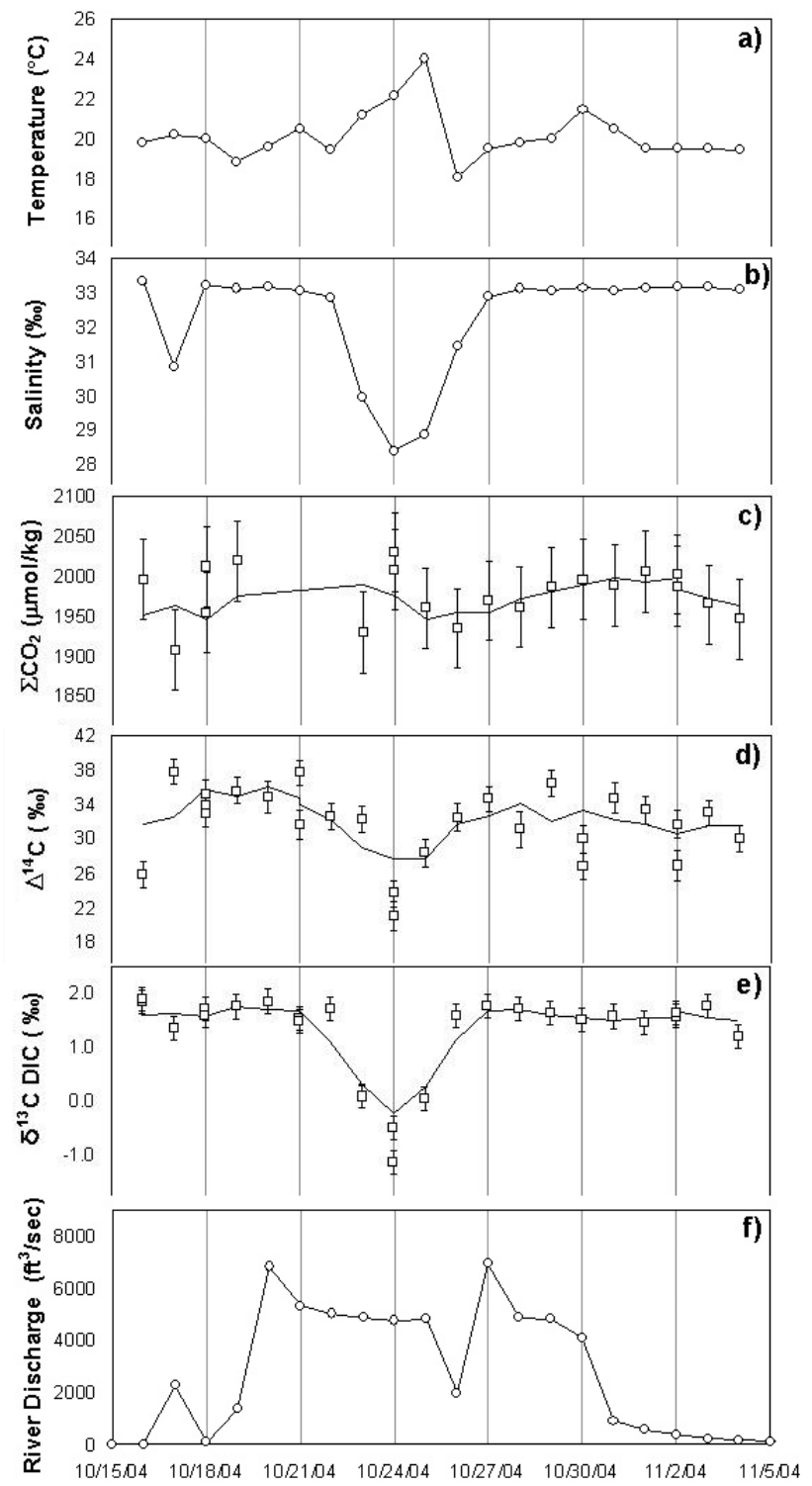

Figure 5 NBP Daily Collection (a) SST, (b) salinity, (c) $\Sigma \mathrm{CO}_{2}$, (d) $\Delta^{14} \mathrm{C}$, (e) $\delta^{13} \mathrm{C}$ and (f) Santa Ana River discharge from 16 October to 11 November 2004. Trend lines for $\Delta^{14} \mathrm{C}, \delta^{13} \mathrm{C}$, and $\Sigma \mathrm{CO}_{2}$ are 3-point moving average determinations.

\section{Santa Ana River Input to the NBP Site}

As mentioned earlier, the Santa Ana River system is one of the largest rivers in Southern California, and its mouth is located about 2 miles northeast of our sampling site (Figure 1). Since we observed admixture of freshwater at our site during the end of 2004, we decided to sample river waters to make isotopic measurements as well. Water samples were collected occasionally from upriver. 
$\Sigma \mathrm{CO}_{2}$ measured in 3 samples of Santa Ana River water $(2960,2566,2734 \mu \mathrm{mol} / \mathrm{kg}$ on 3/25/05, $7 /$ $21 / 05,1 / 11 / 07$, respectively - denoted by SAR in first column of Table 1) were higher than in seawater. The salinity values of the SAR samples are $0.36 \%$, $32.77 \%$, and $24.81 \%$, respectively, indicating that the SAR channel acts as a reverse estuary, changing direction of water flow seasonally.

$\Delta{ }^{14} \mathrm{C}$ results of the SAR samples are $0.8 \%$ (average of 2 points), $42.2 \%$, and $12.2 \%$, on $3 / 25 / 05$, $7 / 21 / 05,1 / 11 / 07$, respectively, when $\delta^{13} \mathrm{C}$ results are $-11.3 \%, 1.8 \%$, and $-4.8 \%$. These values corroborate the presence of seawater in the channel. The low carbon isotopic results suggest that the Santa Ana River can also be a source of low isotopic values to the NBP site. The $\delta^{13} \mathrm{C}$ value from $3 /$ $25 / 05(-11.3 \%)$ is likely a result of remineralization of organic matter that contains low $\delta^{13} \mathrm{C}$ values. Since the beginning of summer 2005 to January 2008, there was little to no precipitation and no significant input of water from the Santa Ana River observed at the NBP site. However, the variability of these 3 proxies (DIC $\Delta^{14} \mathrm{C}$, DIC $\delta^{13} \mathrm{C}$, and salinity) for the few samples measured are in good agreement, and suggests mixing of Santa Ana River water with near-shore ocean water predictably alters the isotopic and salinity signals at the NBP site.

\section{NBP Long-Term Collection}

We present water temperature, $\Sigma \mathrm{CO}_{2}$, DIC $\delta^{13} \mathrm{C}$, DIC $\Delta^{14} \mathrm{C}$, and salinity values, and the Bakun index (Bakun 1973 , from $33^{\circ} \mathrm{N}, 119^{\circ} \mathrm{W}$ ), from $5 / 05$ to $1 / 08$ in Figures $6 \mathrm{a}-\mathrm{f}$. The trend lines represent the 3-point moving averages. While data for all samples are listed in Table 1, only data from samples with salinity values $\geq 33.00 \%$ are shown in Figures $6 \mathrm{a}-\mathrm{f}$ to ensure that the comparisons among these proxies are from marine origin only (i.e. data from 4/6/06, 5/4/06, and 1/11/07 are not plotted in Figure 6). While some $\Delta^{14} \mathrm{C}$ and $\delta^{13} \mathrm{C}$ values between January and May 2005 are not depleted, high precipitation and runoff from the Santa Ana River continued to reach the NBP site during this period, resulting in salinity values consistently lower than $33.00 \%$ ( $24.45-32.85 \%$ ). To maintain consistency, data from the period prior to May 2005 is not included in Figure 6.

The SST values ranged from $13-25^{\circ} \mathrm{C}$ and averaged $18{ }^{\circ} \mathrm{C}$ over the 3 -yr period. Maximum SST values were reached in $7 / 05,7 / 06$, and $8 / 07$ with values of $24^{\circ} \mathrm{C}, 25^{\circ} \mathrm{C}$, and $23^{\circ} \mathrm{C}$, respectively. SST minima occurred in $3 / 06,1 / 07$, and $12 / 07$ with values of $13{ }^{\circ} \mathrm{C}, 14^{\circ} \mathrm{C}$, and $13^{\circ} \mathrm{C}$, respectively (Figure 6a). Total $\mathrm{CO}_{2}$ values averaged $1947 \mu \mathrm{mol} / \mathrm{kg}$ with no significant trend overall, though a minimum in $\Sigma \mathrm{CO}_{2}\left(1743 \mu \mathrm{mol} / \mathrm{kg}\right.$ ) occurred in $8 / 05$ (Figure 6b). A small decrease in $\delta^{13} \mathrm{C}$ DIC values is observed from 2005-2008 ( 0.5\%); values averaged 1.6\%. The sample from $2 / 16 / 06$ had a noticeably high value of $3.3 \%$, while samples from $4 / 6 / 06$ (3 replicates) were noticeably low $(-0.6 \%$ ) (Figure 6c).

No long-term change in DIC $\Delta^{14} \mathrm{C}$ values is observed over the 2005-2006 time series and values averaged 33.7\%. High $\Delta^{14} \mathrm{C}$ values were found for 4/15/05 (see Table 1), 6/27/05, 1/13/06, 8/15/06, and 2/14/07 (65.4\%o, 43.3\%o, 51.0\%, 46.0\%, and 48.4\%o, respectively). In 5/07, DIC $\Delta^{14} \mathrm{C}$ values reached a minimum of $20.9 \%$ (Figure $6 \mathrm{~d}$ ), then increased slightly to $1 / 08$.

Salinity values remained somewhat constant through $11 / 06$, averaging $33.37 \%$. Salinity values steadily increased to $33.80 \%$ by $5 / 07$ and then decreased to $33.42 \%$ by $1 / 08$ (Figure 6e).

The $\Delta^{14} \mathrm{C}$ values of shell samples collected from the NBP site on $05 / 04 / 06$ ( $\sim 6$-month-old mussel and barnacle shells), 1/18/07 ( 6-month-old mussel), and 1/31/08 ( 4- and 5-month-old mussels) yielded $34.8 \%$, 36.3\%o, 32.3\%, 29.1\%o, and 27.4\%o, respectively (asterisks in Table 1). These results are within the ranges of the DIC $\Delta^{14} \mathrm{C}$ values obtained for their individual estimated ages. 


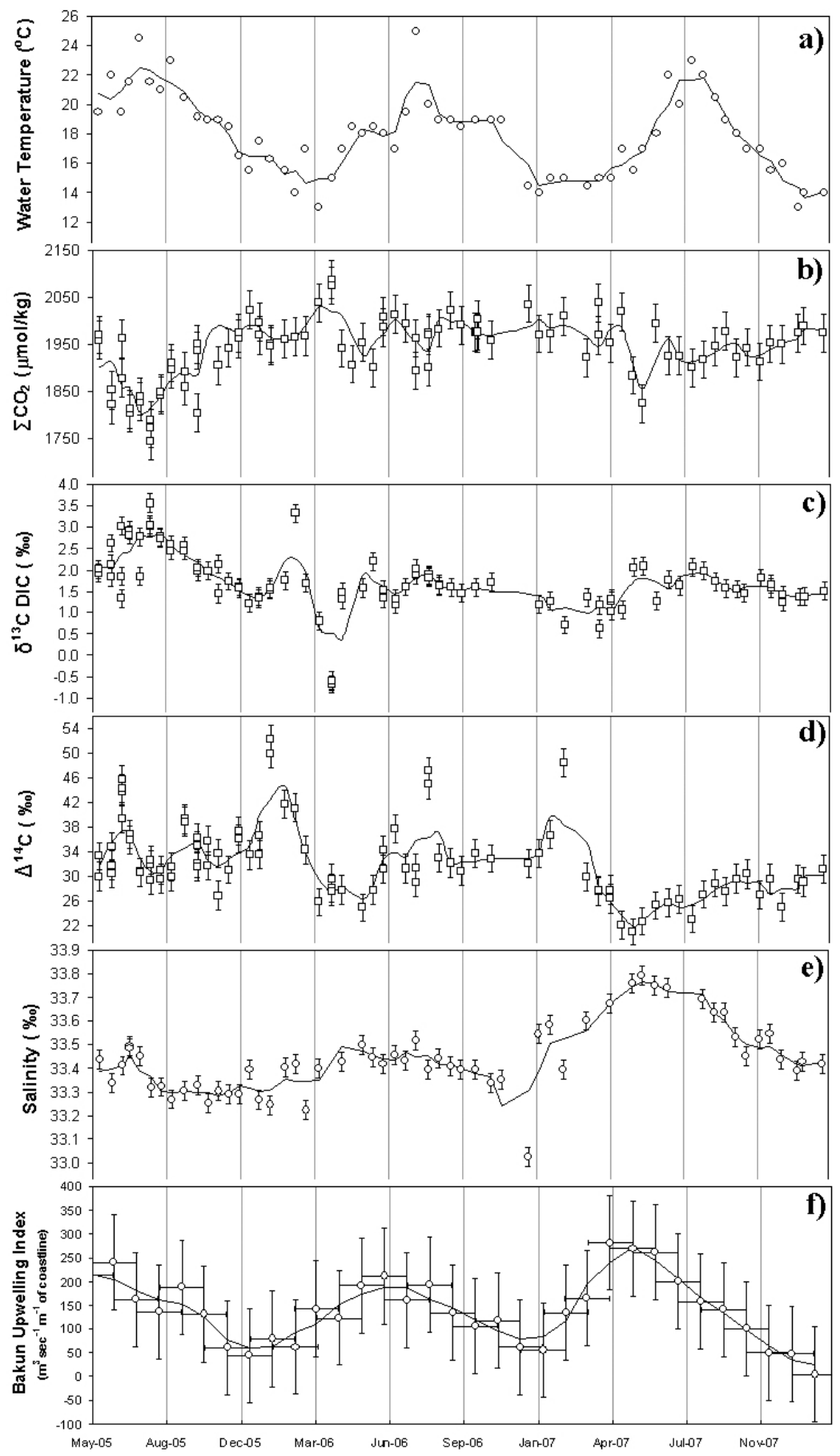

Figure 6 NBP Long Term Collection (a) SST, (b) $\Sigma \mathrm{CO}_{2}$, (c) $\delta^{13} \mathrm{C}$, (d) $\Delta^{14} \mathrm{C}$, (e) salinity measurements of seawater samples from this work, and (f) Bakun index values (Bakun 1973) for $33^{\circ} \mathrm{N}, 119^{\circ} \mathrm{W}$ from 2005 to 2008. Trend lines are 3point moving average determinations. Results from samples with salinity values $<33.00 \%$ were removed to assure that the comparisons among proxies are from marine environmental samples only. The entire data set with all measurements and determinations obtained for this work is in Table 1. 
Killingley and Berger (1979) reported a record of seasonal upwelling from the stable carbon isotope measurements of modern mollusks shells that showed lower $\delta^{13} \mathrm{C}$ values from upwelled water advecting from depth (Kroopnick et al. 1970). Our DIC $\delta^{13} \mathrm{C}$ values show a significant anticorrelation with the $\Sigma \mathrm{CO}_{2}$ measurements $(r=-0.79)$ (Table 3$)$. On $4 / 6 / 06$, a very low DIC $\delta^{13} \mathrm{C}$ value was obtained $(-0.75 \%, n=2$, Table 1$)$. This low $\delta^{13} \mathrm{C}$ value is coincident with a maximum $\Sigma \mathrm{CO}_{2}$ value $(2075 \mu \mathrm{M} / \mathrm{kg}, n=3)$ that may have been caused by decomposition of ${ }^{13} \mathrm{C}$-depleted organic material (Sheu et al. 1996). However, because numerous factors affect DIC $\delta^{13} \mathrm{C}$ values and $\Sigma \mathrm{CO}_{2}$ concentrations, we cannot definitively assign a cause or causes to this correlation.

Table 3 Linear correlation coefficient $r$ for each of the linear correlations of data from monthly averaged data points from NPB $(n=33)$. Correlation is significant when indicated by a significance level in parentheses $(p)$.

\begin{tabular}{lllll}
\hline & $\Delta^{14} \mathrm{C}$ & $\delta^{13} \mathrm{C}$ & $\Sigma \mathrm{CO}_{2}$ & Salinity \\
\hline$\Delta^{14} \mathrm{C}$ & 1 & & & \\
$\delta^{13} \mathrm{C}$ & 0.2 & 1 & & \\
$\Sigma \mathrm{CO}_{2}$ & 0.2 & $-0.791(.001)$ & 1 & \\
Salinity & $-0.53(.002)$ & 0.122 & 0.28 & 1 \\
Bakun & $-0.385(.05)$ & 0.13 & 0.31 & $0.491(.005)$ \\
\hline
\end{tabular}

High $\Delta^{14} \mathrm{C}$ values occurred every 6-7 months from 6/05 through 2/07 (Figure 6d). Using satellite data, DiGiacomo and Holt (2001) determined that small-scale eddies $(<50 \mathrm{~km}$ diameter) frequently occur in the SCB during winter, including a cluster of eddies near the southern coast of San Clemente Island and around Catalina Island ( $45 \mathrm{~km}$ from the NBP). Throughout the rest of the year, eddies are distributed throughout the SCB but in fewer numbers and further apart. The occurrence of nearby eddies could bring open ocean waters to our sampling site, resulting in periodic high $\Delta^{14} \mathrm{C}$ values. Analysis of wind speed and direction revealed no obvious patterns. Eddy formation is a complex process involving not only wind but surface currents and topography. The effect of wind during our NBP Daily Collection was easier to discern because the data was taken daily and the source of low salinity water was clearly indicated (Santa Ana River).

While the chemical and physical parameters obtained by the CALCOFI and IMECOCAL cruises (covering a large area north and seaward of NBP) showed periods of upwelling through 2005 and 2006, our DIC $\Delta^{14} \mathrm{C}$ results and other proxies did not show similar patterns of upwelling at the NBP site (at the coastline). At the beginning of March 2007, however, our DIC $\Delta{ }^{14} \mathrm{C}$ values decreased by about $10 \%$ while salinity values increased by $\sim 0.30 \%$, which can be interpreted as an increase in upwelling of deeper waters to the surface. Salinity and $\Delta^{14} \mathrm{C}$ values gradually returned to near normal by the end of 2007 .

\section{Upwelling Intensity in the Southern California Bight}

The Pacific Fisheries Environmental Laboratory (PFEL) regularly produces the Bakun upwelling index, which estimates the intensity of large-scale, wind-induced coastal upwelling using geostrophic wind approximations (Bakun 1973; Schwing et al. 1996; ftp://orpheus.pfeg.noaa.gov/outgoing/upwell/monthly/upindex.mon). Positive index values indicate upwelling while negative values indicate downwelling. Pickett and Schwing (2006) have evaluated these upwelling estimates using high-resolution satellite wind measurements combined with a global atmospheric model, and concluded that the index is reasonably accurate for the North American west coast. 
Figure 7 displays the annual maxima of the Bakun index data from the area closest to our station from 1974 to 2007. It shows that the second half of the Bakun index (1991-2007) is significantly lower than the first half $(1974-1990)(p<0.05)$, indicating a trend toward reduced coastal upwelling. This reduction may be associated with the warming of the surface ocean during that period, which would trigger a decrease in wind stress as hypothesized by Gucinski et al. (1990). Roemmich and McGowan (1995) attributed the $70 \%$ decline of zooplankton populations observed in the CCS to warming of surface waters. They concluded that higher sea-surface temperatures can lead to increased stratification, a shallower mixed layer, and reduced mixing with deeper water (decreased upwelling), followed by reduced replenishment of nutrients needed for photosynthesis (McGowan et al. 1998).

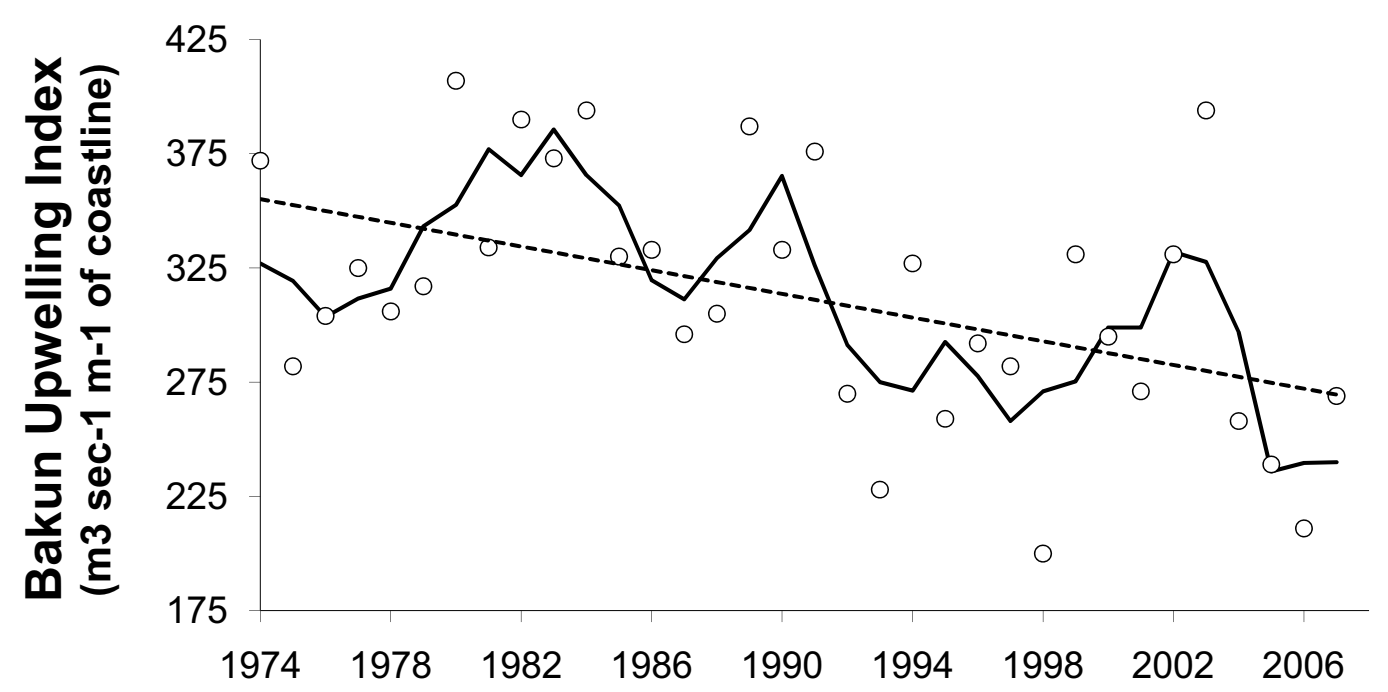

Figure 7 Annual peak maximums in the Bakun upwelling index for coastal Orange County, California $\left(33^{\circ} \mathrm{N}, 119^{\circ} \mathrm{W}\right)$. On plot: (O) Bakun data points close to our station for the last $33 \mathrm{yr}$ (1974-2007), (-) 3-yr moving average, and (---) linear trend line showing a significant decrease in the upwelling signal with time.

Least-squares fits (Type II) were performed between each of the 5 monthly averaged (bimonthly values were averaged) data sets (Bakun index, $\Delta^{14} \mathrm{C}, \delta^{13} \mathrm{C}, \Sigma \mathrm{CO}_{2}$, and salinity, $n=33$ ) from the NBP long time series, and the results are shown in Table 3. Correlation is significant to at least the $95 \%$ level $(p=0.05)$ for 4 of the least-squares fits: $\Sigma \mathrm{CO}_{2}$ versus $\delta^{13} \mathrm{C}(r=-0.791, p=0.001)$; salinity versus $\Delta^{14} \mathrm{C}(r=-0.530, p=0.002)$; salinity versus Bakun index $(r=0.491, p=0.005)$; and $\Delta^{14} \mathrm{C}$ versus Bakun index $(r=-0.385, p=0.05)$. These results confirm that there is a relationship between upwelling in the SCB (Bakun index) and 2 of our measured quantities, $\Delta^{14} \mathrm{C}$ and salinity. During times when $\Delta^{14} \mathrm{C}$ is low and salinity values are high, the level of upwelling in this region is generally high. In contrast, when $\Delta^{14} \mathrm{C}$ is high and salinity values are low, the level of upwelling is lower than normal. This suggests that $\Delta^{14} \mathrm{C}$ in water from the NBP is correlated with upwelling in the SCB.

Regarding surface $\Delta^{14} \mathrm{C}$ variations as a tracer of upwelling strength, earlier observations in Half Moon Bay (Robinson 1981) show large seasonal changes. However, these changes are attributed to the enhanced upwelling in this northern California coastal region and to the larger gradient between atmosphere and surface ocean waters during the period of sample collection (1978-1979). In southern California, surface DIC $\Delta^{14} \mathrm{C}$ values $(<30 \mathrm{~m})$ had decreased systematically from 1973 to 2004 (Figure 8). The lack of a discernible gradient between the $\Delta^{14} \mathrm{C}$ in surface $(<30 \mathrm{~m})$ and subsurface 
(85-200 m) waters since the mid-1990s is associated in part due to the delayed penetration of bomb $\Delta^{14} \mathrm{C}$ into the subsurface waters of the northeast Pacific (Key et al. 1996). Although there appears to be no difference between the surface $\Delta^{14} \mathrm{C}$ values and those in upwelled subsurface waters in the $\mathrm{SCB}$, our data illustrate the subtlety of the $\Delta^{14} \mathrm{C}$ difference between the 2 water masses when this parameter was correlated with other proxies. The DIC $\Delta^{14} \mathrm{C}$ minimum/salinity maximum during 2007 corresponds with an increase in the Bakun index for this period (Figure 6d-f), and coincides with the transition to a La Niña phase in the Pacific Ocean in mid-2007. Note that the $\Delta^{14} \mathrm{C}$ historical data for the upper $200 \mathrm{~m}$ (Figure 8) is quite sparse and from a relatively large area of the northeast Pacific. Since we know of no other data sets to compare with our results, this limits the conclusions that can be made regarding the causal link between recent $\Delta^{14} \mathrm{C}$ measurements and upwelling strength in the SCB.

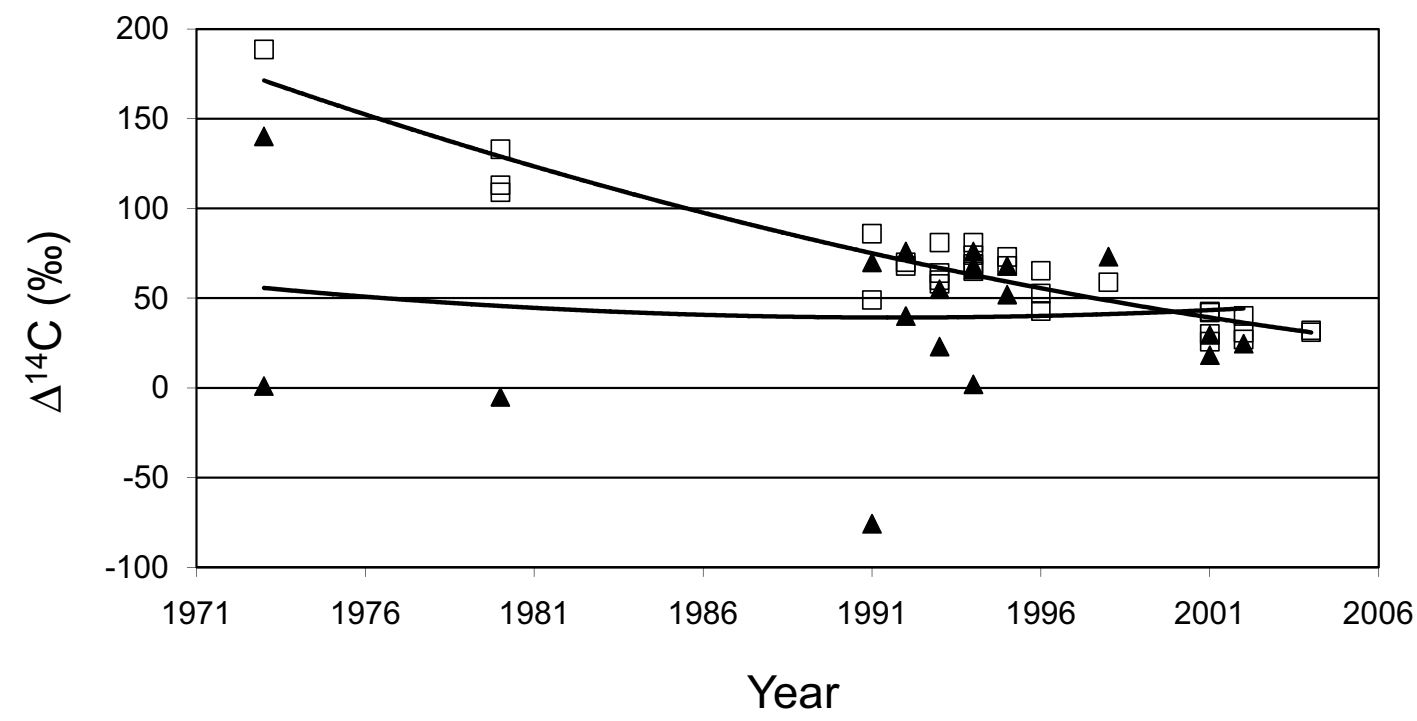

Figure 8 Historical $\Delta^{14} \mathrm{C}$ values for the Southern California region $\left(27^{\circ} 33^{\prime} \mathrm{N}\right.$ to $34^{\circ} 50^{\prime} \mathrm{N}$, and $114^{\circ} 52^{\prime} \mathrm{W}$ to $\left.127^{\circ} 53^{\prime} \mathrm{W}\right)$ from 1973 to 2004 (Östlund and Stuiver 1980; Druffel and Williams 1991; Key et al. 1996; Masiello et al. 1998; Beaupré 2007). Squares $(\square)$ are for samples from depths $0-30 \mathrm{~m}$ and filled triangles $(\boldsymbol{\Delta})$ are for depths $85-200 \mathrm{~m}$.

In this work, we demonstrate the importance of combining several proxies with meteorological data, to understand the onset and duration of recent coastal upwelling at a site in the SCB. Seawater sampling at our NBP site is ongoing and future work will involve sampling at offshore sites. We also intend to expand our study of mollusk shells at the NBP site and other sites along the California coast.

\section{ACKNOWLEDGMENTS}

We thank Rachel B Moore and Robert Beverly for help with sample preparation, and the Scripps ODF group for the salinity measurements. We also thank Ann McNichol and one anonymous reviewer for their very thoughtful comments and recommendations. We acknowledge the $\mathrm{W} M$ Keck Foundation, NSF Chemical Oceanography Program and UCI for financial support. 


\section{REFERENCES}

Bakun A. 1973. Coastal upwelling indices, west coast of North America, 1946-71. US Department of Commerce, NOAA Technical Report, NMFS SSRF-671.

Beaupré SR. 2007. Dissolved organic carbon concentrations and isotope ratios in the northeast Pacific Ocean [PhD dissertation]. Earth System Science Department, University of California, Irvine. $142 \mathrm{p}$.

Bray NA, Keyes A, Morawitz WML. 1991. The California Current system in the Southern California Bight and the Santa Barbara Channel. Journal of Geophysical Research 104(C4):7695-714.

Coe WR, Fox DL. 1942. Biology of the California seamussel (Mytilus Californianus). I. Influence of temperature, food supply, sex and age on the rate of growth. Journal of Experimental Zoology 90(1):1-30.

DiGiacomo PM, Holt B. 2001. Satellite observations of small coastal ocean eddies in the Southern California Bight. Journal Geophysical Research 106(C10): 22,521-43.

Druffel ERM, Williams PM. 1991. Radiocarbon in seawater and organisms from the Pacific coast of Baja California. Radiocarbon 33(3):291-6.

Goericke R, Venrick E, Koslow T, Sydeman WJ, Schwing FB, Bograd SJ, Peterson WT, Emmett R, Rubén LLJ, Castro GG, Valdez JG, Hyrenbach KD, Bradley RW, Weise MJ, Harvey JT, Collins C, Lo NCH. 2007. The state of the California Current, 20062007: regional and local processes dominate. California Cooperative Oceanic Fisheries Investigations Report 48:33-66.

Gucinski HRT, Lackey RT, Spence BC. 1990. Global climate change: policy implications for fisheries. Fisheries 15(6):33-8.

Guilderson T, Roark EB, Quay PD, Flood Page SR, Moy C. 2006. Seawater radiocarbon evolution in the Gulf of Alaska: 2002 observations. Radiocarbon 48(1):115

Hutchings L, Pitcher GC, Probyn TA, Bailey GW. 1995. The chemical and biological consequences of coastal upwelling. In: Summerhayes CP, Emeis KC, Angel MV, Smith RL, Zeitzschel B, editors. Upwelling in the Ocean: Modern Processes and Ancient Records. Environmental Science Research Report ES 18. New York: Wiley. p 65-83.

Key RM, Quay PD, Jones GA, Schneider RJ, McNichol AP, von Reden K, Schneider RJ. 1996. WOCE AMS radiocarbon I: Pacific Ocean results (P6, P16, and P17). Radiocarbon 38(3):425-518.

Killingley JS, Berger WH. 1979. Stable isotopes in a mollusk shell: detection of upwelling events. Science 205(4402):186-8.

Kroopnick P, Deuser WG, Craig H. 1970. Carbon 13 measurements on dissolved inorganic carbon at the North Pacific (1969) Geosecs Station. Journal Geophysical Research 75(36):7668-71.
McGowan JA, Cayan DR, Dorman LM. 1998. Climateocean variability and ecosystem response in the Northeast Pacific. Science 281(5374):210-7.

Masiello CA, Druffel ERM, Bauer JE. 1998. Physical controls on dissolved inorganic radiocarbon variability in the California Current. Deep-Sea Research II 45(4-5):617-42.

McNaught AD, Wilkinson A. 1997. Compendium of Chemical Terminology. 2nd edition. Oxford: Blackwell Scientific.

McNichol AP, Jones GA. 1991. Measuring ${ }^{14} \mathrm{C}$ in seawater $\Sigma \mathrm{CO}_{2}$ by accelerator mass spectrometry, WHP operations and methods. In: Joyce T, Corry C, Stalcup M, editors. WOCE Operations and Methods Manual. Woods Hole, Massachusetts: WHPO Publication 90$1: 71$.

McNichol AP, Jones GA, Hutton DL, Gagnon AR. 1994. The rapid preparation of seawater $\Sigma \mathrm{CO}_{2}$ for radiocarbon analysis at the National Ocean Sciences AMS Facility. Radiocarbon 36(2):237-46.

Östlund HG, Stuiver M. 1980. GEOSECS Pacific radiocarbon. Radiocarbon 22(1):25-53.

Panel on the Southern California Bight of the Committee on a Systems Assessment of Marine Environmental Monitoring (PSCB). 1990. Monitoring Southern California's Coastal Waters. National Research Council (U.S.): Commission on Engineering and Technical Systems. Washington, DC: National Academy Press, Marine Board.

Peterson B, Emmett R, Goerlicke R, Venrick E, Mantyla A, Bogard S, Schwing F, Ralston S, Forney K, Hewitt R, Lo N, Watson W, Barlow J, Lowry M, Lavaniegos BE, Chavez F, Sydeman WJ, Hyrenbach D, Bradley RW, Warzybok P, Hunter K, Benson S, Weise M, Harvey JT. 2006. The state of the California Current, 2005-2006: Warm in the north, cool in the south. California Cooperative Oceanographic Fisheries Investigation Data Report 47:30-75.

Pickett MH, Schwing FB. 2006. Evaluating upwelling estimates off the west coasts of North and South America. Fisheries Oceanography 15(3):256-9.

Robinson SW. 1981. Natural and man-made radiocarbon as a tracer for coastal upwelling processes. In: Richards FA, editor. Coastal Upwelling. Washington, DC: American Geophysical Union. p 298-302.

Roemmich D, McGowan J. 1995. Climatic warming and the decline of zooplankton in the California Current. Science 267(5202):1324-6.

Santos GM, Southon JR, Druffel-Rodriguez KC, Griffin S, Mazon M. 2004. Magnesium perchlorate as an alternative water trap in AMS graphite sample preparation: a report on sample preparation at the KCCAMS Facility at the University of California, Irvine. Radiocarbon 46(1):165-73.

Santos GM, Moore RB, Southon JR, Griffin S, Hinger E, 
Zhang D. 2007. AMS ${ }^{14} \mathrm{C}$ sample preparation at the KCCAMS/UCI Facility: status report and performance of small samples. Radiocarbon 49(2):255-69.

Schwing FB, O’Farrell M, Steger JM, Baltz KA. 1996. Coastal upwelling indices, west coast of North America, 1946-95. US Department of Commerce. NOAATM-NMFS-SWFSC-231. p 1-32.

Scott ME, Boaretto E, Bryant C, Cook GT, Gulliksen S, Harkness DD, Heinemeier J, McGee E, Naysmith P, Possnert G, van der Plicht H, van Strydonck M. 2004. Future needs and requirements for $\mathrm{AMS}{ }^{14} \mathrm{C}$ standards and reference materials. Nuclear Instruments and
Methods in Physics Research B 223-224:382-7.

Sheu DD, Lee WY, Wang CH, Wei CL, Chen CTA, Cherng C, Huang MH. 1996. Depth distribution of $\delta^{13} \mathrm{C}$ of dissolved $\Sigma \mathrm{CO}_{2}$ in seawater off eastern Taiwan: effects of Kuroshio current and its associated upwelling phenomenon. Continental Shelf Research 16(12):1609-19.

Stuvier M, Polach H. 1977. Discussion: reporting of ${ }^{14} \mathrm{C}$ data. Radiocarbon 19(3):355-63.

Tsuchiya M. 1975. California Undercurrent in the Southern California Bight. California Cooperative Oceanographic Fisheries Investigation 18:155-8. 